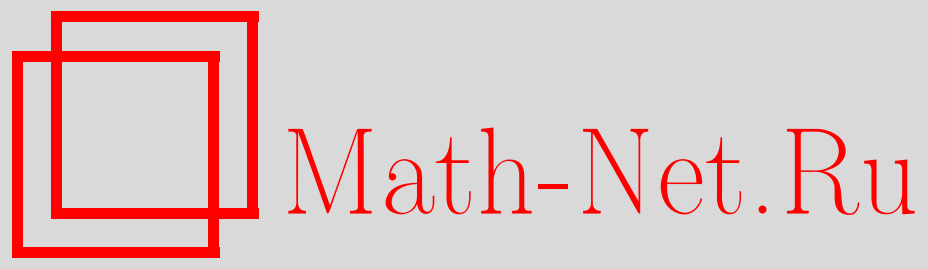

Я. Варшавский, Д. А. Каждан, Лемма Йонеды для полных пространств Сигала, Функи. анализ и его прил., 2014, том 48, выпуск 2, 3-38

DOI: https://doi.org/10.4213/faa3147

Использование Общероссийского математического портала MathNet.Ru подразумевает, что вы прочитали и согласны с пользовательским соглашением http://www. mathnet.ru/rus/agreement

Параметры загрузки:

IP : 3.85 .5 .30

26 апреля 2023 г., 12:12:18

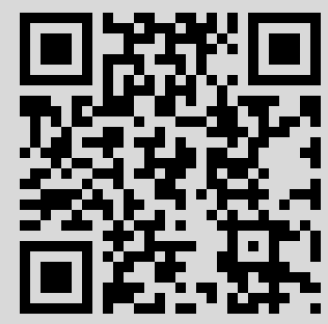


Функционалъный анализ и его приложения

2014, т. 48, вып. 2, с. 3-38

УДК $512.58+515.14$

\title{
Лемма Йонеды для полных пространств Сигала*
}

\author{
(c) 2014. Я. ВАРШАВСКИЙ, Д. КАЖДАН
}

Памяти И. М. Гельфанда

В заметке дана формулировка и замкнутое доказательство леммы Йонеды для $\infty$-категорий на языке полных пространств Сигала.

\section{Введение}

В последние годы $\infty$-категории, или, более формально, $(\infty, 1)$-категории, возникают в различных областях математики. Например, они стали неотъемлемым ингредиентом геометрической проблемы Ленглендса. В книгах [4], [5] Лурье разработал теорию $\infty$-категорий на языке квазикатегорий и распространил на этот случай многие результаты обычной теории категорий.

Резк [6] ввел другую модель $\infty$-категорий, которую он назвал полными пространствами Сигала. Эта модель имеет определенные преимущества. Например, ее можно обобщить на $(\infty, n)$-категории (см. [7]).

Естественно было бы распространить результаты обычной теории категорий на случай полных пространств Сигала. В настоящей статье это сделано для леммы Йонеды.

Чтобы сформулировать ее, нам нужно построить удобную модель « $\infty$-категории пространств», которая была бы $\infty$-аналогом категории множеств. Под влиянием результатов Лурье мы определяем эту $\infty$-категорию как симплициальное пространство, «классифицирующее левые расслоения». После того как это сделано, построение вложения Йонеды и доказательство леммы Йонеды проводятся почти таким же образом, как и в случае обычных категорий.

В последующих статьях [2], [3] мы изучаем сопряженные функторы, пределы и копределы. Мы докажем более сильную версию леммы Йонеды и обобщим результаты данной работы на $(\infty, n)$-категории.

Мы благодарны Эммануэлю Фарджуну, Владимиру Хиничу и Нику Розенблюму за воодушевляющие беседы и ценные замечания.

Эта работа устроена следующим образом. Чтобы сделать изложение замкнутым, в §1 мы приводим основные определения и обсуждаем свойства модельных категорий, симплициальных множеств, симплициальных пространств и пространств Сигала, предполагая известными лишь основы теории категорий. В 22 мы вводим левые расслоения, строим $\infty$-категорию пространств $\mathfrak{S}$ и приводим формулировку и доказательство леммы Йонеды. В $\S 3$ изучаются квазирасслоения симплициальных пространств, которые необходимы для наших рассуждений, а также сами по себе являются весьма интересными объектами. Последний

* Исследования Я. В. частично поддержаны грантом ISF №1017/13, исследования Д. К. частично поддержаны грантом ERC №247049-GLC. 
параграф посвящен доказательству свойств $\infty$-категории $\mathfrak{S}$, сформулированных во втором параграфе.

\section{§1. Предварительные сведения}

\section{1. Модельные категории.}

1.1.1. Обозначения. Пусть $\mathscr{C}$ - категория. (а) Для объекта $Z \in \mathscr{C}$ обозначим через $\mathscr{C} / Z$ надкатегорию над $Z$, т. е. категорию морфизмов $X \rightarrow Z$ в $\mathscr{C}$.

(b) Для пары морфизмов $i: A \rightarrow B$ и $p: X \rightarrow Y$ в $\mathscr{C}$ обозначим через $\operatorname{Hom}_{\mathscr{C}}(i, p)$ множество коммутативных диаграмм в $\mathscr{C}$ вида

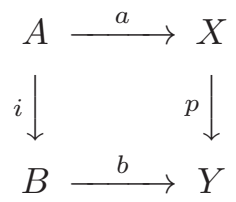

Будем говорить, что $i-$ ретракт морфизма $p$, если существуют $\alpha \in \operatorname{Hom}_{\mathscr{C}}(i, p)$ и $\beta \in \operatorname{Hom}_{\mathscr{C}}(p, i)$, такие, что $\beta$ ○ $\alpha=\operatorname{Id}_{i}$.

(c) Будем говорить, что $p$ обладает свойством правой поднимаемости или, короче, поднимаем справа относительно $i$ (и что $i$ обладает свойством левой поднимаемости или, короче, поднимаем слева относительно $p$ ), если для любой коммутативной диаграммы (1.1) существует морфизм $c: B \rightarrow X$, такой, что $p \circ c=b$ и $c \circ i=a$.

Очевидно, это происходит тогда и только тогда, когда естественное отображение множеств

$$
\left(i^{*}, p_{*}\right): \operatorname{Hom}(B, X) \rightarrow \operatorname{Hom}(A, X) \times_{\operatorname{Hom}(A, Y)} \operatorname{Hom}(B, Y)
$$

сюръективно.

(d) Предположим, что в $\mathscr{C}$ существуют расслоенные произведения. Тогда для морфизма $f: X \rightarrow Y$ в $\mathscr{C} / Z$ и морфизма $g: Z^{\prime} \rightarrow Z$ в $\mathscr{C}$ будем писать $g^{*}(f): g^{*}(X) \rightarrow g^{*}(Y)$ вместо $f \times_{Z} Z^{\prime}: X \times_{Z} Z^{\prime} \rightarrow Y \times_{Z} Z^{\prime}$ и назовем этот морфизм пулбэком морфизма $f$.

(е) Будем говорить, что категория $\mathscr{C}$ декартова, если в ней существуют конечные произведения и для любых $X, Y \in \mathscr{C}$ существует элемент $X^{Y} \in \mathscr{C}$, представляющий функтор $Z \mapsto \operatorname{Hom}(Z \times Y, X)$; этот элемент называется внутренним hom элементов $X$ и $Y$.

1.1.2. Пример. Пусть $\mathscr{C}$ - категория функторов, $\mathscr{C}=\operatorname{Fun}\left(\Gamma^{o p}, S e t\right)$, где $\Gamma-$ малая категория, а Set-категория множеств.

(a) Так как в категории Set существуют все пределы и копределы, то это же верно и для категории $\mathscr{C}$. Явным образом, функтор $\alpha: I \rightarrow \mathscr{C}$ для каждого $\gamma \in \Gamma$ определяет функтор $\alpha(\gamma): I \rightarrow S e t$, такой, что $\left(\lim _{I} \alpha\right)(\gamma)=\lim _{I}(\alpha(\gamma))$ и аналогичное соотношение выполнено для копределов. В частности, в категории $\mathscr{C}$ существуют произведения.

(b) Для любого $\gamma \in \Gamma$ обозначим через $F_{\gamma} \in \mathscr{C}$ представимый функтор $\operatorname{Hom}_{\Gamma}(\cdot, \gamma)$. Тогда для любых $X, Y \in \mathscr{C}$ существует внутренний hom $X^{Y} \in \mathscr{C}$, определенный правилом $X^{Y}(\gamma)=\operatorname{Hom}\left(Y \times F_{\gamma}, X\right)$ с очевидными отображениями перехода. Другими словами, категория $\mathscr{C}$ декартова.

Следующая лемма непосредственно вытекает из определений. 
1.1.3. Лемма. Пусть $\mathscr{C}$ - декартова категория, и пусть $i: A \rightarrow B, j: A^{\prime} \rightarrow$ $B^{\prime}$ и $p: X \rightarrow Y-$ морфизмы в $\mathscr{C}$. Тогда $j$ поднимаем слева относительно $\left(i^{*}, p_{*}\right): X^{B} \rightarrow X^{A} \times_{Y^{A}} Y^{B}$ в том и только в том случае, когда морфизм $\left(i_{*}, j_{*}\right):\left(A \times B^{\prime}\right) \sqcup_{\left(A \times A^{\prime}\right)}\left(B \times A^{\prime}\right) \rightarrow B \times B^{\prime}$ поднимаем слева относительно $p$.

1.1.4. Определение (ср. [1, гл. II, §1]). Модельная категория - это категория $\mathscr{C}$, оснащенная тремя наборами морфизмов, называемых корасслоениями, расслоениями и слабыми эквивалентностями соответственно, которая удовлетворяет следующим аксиомам:

CM1. В категории $\mathscr{C}$ существуют все конечные пределы и копределы.

CM2 (свойство «2 из 3»). Если в диаграмме $X \stackrel{f}{\rightarrow} Y \stackrel{g}{\rightarrow} Z$ какие-либо два из морфизмов $f, g$ и $g \circ f$ являются слабыми эквивалентностями, то третий морфизм также является слабой эквивалентностью.

СМ3 (ретракт). Если морфизм $f$ является ретрактом морфизма $g$, а $g-$ слабая эквивалентность, расслоение или корасслоение, то $f$ также обладает соответствующим свойством.

CM4 (свойство поднимаемости). Пусть $i$ - корасслоение, а $p$ - расслоение. Тогда $p$ поднимаемо справа относительно $i$ при условии, что либо $i$, либо $p-$ слабая эквивалентность.

CM5 (свойство разложимости). Любой морфизм $f$ имеет разложения вида

(a) $f=p \circ i$, где $p$ - расслоение, а $i$-корасслоение и слабая эквивалентность;

(b) $f=q \circ j$, где $q$ - расслоение и слабая эквивалентность, а $j$ - корасслоение.

1.1.5. Обозначения. (а) Морфизм в модельной категории $\mathscr{C}$ называется тривиальным корасслоением (соответственно тривиальным расслоением), если он является корасслоением (соответственно расслоением) и слабой эквивалентностью.

(b) Говорят, что элемент $X \in \mathscr{C}$ фибрантен (соответственно кофибрантен), если каноническое отображение $X \rightarrow \mathrm{pt}$ (соответственно $\emptyset \rightarrow X)$, где pt (соответственно $\emptyset$ ) - конечный (соответственно начальный) объект категории $\mathscr{C}$, является расслоением (соответственно корасслоением).

(c) Согласно аксиоме CM5, каждый морфизм $f: X \rightarrow Y$ можно записать в виде композиции $X \stackrel{i}{\rightarrow} X^{\prime} \stackrel{p}{\rightarrow} Y$, где $i$ - тривиальное корасслоение, а $p-$ расслоение. В этом случае говорят, что $p$ - фибрантная замена морфизма $f$. В частности, если $Y=\mathrm{pt}$, то говорят, что $X^{\prime}-$ фибрантная замена объекта $X$.

Следующий фундаментальный факт содержится, например, в [1, гл. II, лемма 1.1].

1.1.6. Лемма. (а) Отображение $f: X \rightarrow Y$ в модельной категории $\mathscr{C}$ является корасслоением (соответственно тривиальным корасслоением) тогда и только тогда, когда оно поднимаемо слева относительно всех тривиальных расслоений (соответственно расслоений).

(b) Отображение $f: X \rightarrow Y$ в модельной категории $\mathscr{C}$ является расслоением (соответственно тривиальным расслоением) тогда и только тогда, когда оно поднимаемо справа относительно всех тривиальных корасслоений (соответственно корасслоений).

1.1.7. Замечания. (а) Из леммы 1.1.6 вытекает, в частности, что класс (тривиальных) корасслоений и класс (тривиальных) расслоений замкнуты относительно композиций и что все изоморфизмы являются тривиальными корасслоениями и тривиальными расслоениями. 
(b) Из леммы 1.1.6 также сразу следует, что (тривиальные) расслоения сохраняются при всех пулбэках, а (тривиальные) корасслоения сохраняются при всех пушаутах.

(c) Из аксиом CM5(а) и СM2 следует, что всякая слабая эквивалентность $f$ имеет разложение вида $f=p \circ i$, где $p$-тривиальное расслоение, а $i$-тривиальное корасслоение.

1.1.8. Определение. Будем говорить, что модельная категория $\mathscr{C}$ декартова, если она декартова как категория (см. 1.1.1(е)), ее конечный объект кофибрантен и для каждых корасслоения $i: A \rightarrow B$ и расслоения $p: X \rightarrow Y$ индуцированное отображение $q: X^{B} \rightarrow X^{A} \times_{Y^{A}} Y^{B}$ является расслоением и, кроме того, слабой эквивалентностью при условии, что слабой эквивалентностью является $i$ либо $p$.

1.1.9. Замечание. Взяв $A=\emptyset$ или $Y=$ pt в определении декартовой модельной категории, мы получаем следующие частные случаи:

(а) Если $B$ кофибрантно, то для любого (тривиального) расслоения $X \rightarrow Y$ индуцированное отображение $X^{B} \rightarrow Y^{B}$ - (тривиальное) расслоение.

(b) Если $X$ фибрантно, то для любого (тривиального) корасслоения $A \rightarrow B$ индуцированное отображение $X^{B} \rightarrow X^{A}$ - (тривиальное) расслоение.

1.1.10. Лемма. Пусть $\mathscr{C}$ - модельная категория, являющаяся декартовой как категория и такая, что ее конечный обгект кофибрантен. Тогда $\mathscr{C}$ является декартовой модельной категорией в том и только в том случае, когда для любых двух корасслоений $i: A \rightarrow B u i^{\prime}: A^{\prime} \rightarrow B^{\prime}$ индуиированный морбизм $j:\left(A \times B^{\prime}\right) \sqcup_{\left(A \times A^{\prime}\right)}\left(B \times A^{\prime}\right) \rightarrow B \times B^{\prime}$ является корасслоением и, кроме того, слабой эквивалентностью при условии, что слабой эквивалентностью является либо $i$, либо $i^{\prime}$.

Доказательство следует из комбинации лемм 1.1.6 и 1.1.3.

1.1.11. Определение. Модельная категория $\mathscr{C}$ называется

(а) правильной справа, если слабые эквивалентности сохраняются при пулбэках вдоль расслоений;

(b) правилъной слева, если слабые эквивалентности сохраняются при пушаутах вдоль корасслоений;

(с) правильной, если она правильна и слева, и справа.

\section{2. Симплициальные множества.}

1.2.1. Категория $\boldsymbol{\Delta}$. (а) Для $n \geqslant 0$ обозначим через $[n]$ категорию, соответствующую частично упорядоченному множеству $\{0<1<\cdots<n\}$. Пусть $\Delta-$ полная подкатегория малой категории $C a t$, состоящая из объектов $[n], n \geqslant 0$.

(b) Для каждого упорядоченного набора из $m+1$ целых чисел $0 \leqslant k_{0} \leqslant$ $k_{1} \leqslant \cdots \leqslant k_{m} \leqslant n$ определим отображение $\delta^{k_{0}, \ldots, k_{m}}:[m] \rightarrow[n]$, такое, что $\delta^{k_{0}, \ldots, k_{m}}(i)=k_{i}$ для всех $i$.

(с) Для $0 \leqslant i \leqslant n$ определим включение $d^{i}:[n-1] \hookrightarrow[n]$, такое, что $i \notin$ $\operatorname{Im} d^{i}$; для $0 \leqslant i<j \leqslant n$ определим включение $d^{i, j}:[n-2] \hookrightarrow[n]$, такое, что $i, j \notin \operatorname{Im} d^{i, j} ;$ для $0 \leqslant i \leqslant n-m$ определим включение $e^{i}:[m] \rightarrow[n]$ формулой $e^{i}(k):=k+i$.

1.2.2. Пространства. (а) Под категорией пространств, или, что то же самое, категорией симплиииальных множеств, мы понимаем категорию функторов $S p:=\operatorname{Fun}\left(\Delta^{o p}, S e t\right)$. 
(b) Для $X \in S p$ положим $X_{n}:=X([n])$. Для любого морфизма $\tau:[n] \rightarrow[m]$ в $\Delta$ обозначим через $\tau^{*}: X_{m} \rightarrow X_{n}$ индуцированное отображение множеств. Для любого морфизма $f: X \rightarrow Y$ в $S p$ обозначим через $f_{n}$ соответствующее отображение $X_{n} \rightarrow Y_{n}$.

(c) Согласно 1.1.2, категория $S p$ декартова и в ней существуют все пределы и копределы.

1.2.3. Стандартный $\boldsymbol{n}$-симплекс. (а) Для каждого $n \geqslant 0$ обозначим через $\Delta[n] \in S p$ функтор $\operatorname{Hom}_{\Delta}(\cdot,[n]): \Delta^{o p} \rightarrow$ Set. Тогда pt $:=\Delta[0]-$ конечный объект категории $S p$.

(b) Лемма Йонеды определяет отождествления $\operatorname{Hom}_{S p}(\Delta[n], X)=X_{n}$ и $\operatorname{Hom}_{S p}(\Delta[n], \Delta[m])=\operatorname{Hom}_{\Delta}([n],[m])$ для всех $X \in S p$ и $n, m \geqslant 0$.

(c) Обозначим через $\Delta^{i}[n]$ образ включения $d^{i}: \Delta[n-1] \rightarrow \Delta[n]$ и положим $\partial \Delta[n]:=\bigcup_{i=0}^{n} \Delta^{i}[n] \subset \Delta[n]$ и $\Lambda^{k}[n]:=\bigcup_{i \neq k} \Delta^{i}[n] \subset \Delta[n]$ для всех $k=0, \ldots, n$.

1.2.4. Слои. (а) Для $X \in S p$ пишем $x \in X$ вместо $x \in X_{0}$. Согласно 1.2.3(b), каждому $x \in X$ соответствует отображение $x: \mathrm{pt} \rightarrow X$.

(b) Для каждого морфизма $f: Y \rightarrow X$ обозначим через $f^{-1}(x)$ или $Y_{x}$ расслоенное произведение $\{x\} \times_{X} Y:=\operatorname{pt} \times_{x, X} Y$ и назовем его слоем морфизма $f$ в точке $x$.

(c) Для любых $Z \in S p$ и $X, Y \in S p / Z$ обозначим через $\operatorname{Map}_{Z}(X, Y)$ слой морфизма $Y^{X} \rightarrow Z^{X}$ над проекцией $(X \rightarrow Z) \in Z^{X}$.

1.2.5. Определение. (а) Отображение $f: X \rightarrow Y$ в $S p$ называется расслоением (Кана), если оно поднимаемо справа относительно включений $\Lambda^{k}[n] \hookrightarrow$ $\Delta[n]$ для всех $n>0, k=0, \ldots, n$.

(b) Отображение $f: X \rightarrow Y$ в $S p$ называется слабой эквивалентностью, если оно индуцирует слабую эквивалентность $|f|:|X| \rightarrow|Y|$ геометрических реализаций (см. [1, с. 60]).

(с) Отображение $f: X \rightarrow Y$ в $S p$ называется корасслоением, если $f_{n}: X_{n} \rightarrow$ $Y_{n}$ является включением для всех $n$.

1.2.6. Теорема. Категория $S p$ имеет структуру правильной декартовой модельной категории, в которой корасслоения, расслоения и слабые эквивалентности заданы определением 1.2.5. В частности, все $X \in S p$ кофибрантны, а тривиальные расслоения - это в точности отображения, поднимаемые справа относительно включений $\partial \Delta[n] \hookrightarrow \Delta[n], n \geqslant 0$.

Доказательство. См. [1, гл. I, теорема 11.3, предложение 11.5, и гл. II, следствие 8.6] с учетом того факта, что в случае модельной категории $S p$ прилагательное «декартова» означает то же самое, что и «симплициальная».

1.2.7. Определение. Будем говорить, что $X \in S p-($ стягиваемый $)$ комплекс Кана, если проекция $X \rightarrow \mathrm{pt}-$ (тривиальное) расслоение.

1.2.8. Компоненты связности. (а) Будем говорить, что объект $X \in S p$ свлзен, если его нельзя представить как несвязное объединение $X=X^{\prime} \sqcup X^{\prime \prime}$, где $X^{\prime}, X^{\prime \prime} \neq \emptyset$. Будем говорить, что $Y \subset X-$ компонента свлзности пространства $X$, если она является максимальным связным подпространством в $X$. Заметим, что $X$ является несвязным объединением своих компонент связности.

(b) Обозначим множество компонент связности пространства $X$ через $\pi_{0}(X)$. Тогда каждое отображение $f: X \rightarrow Y$ в $S p$ индуцирует отображение $\pi_{0}(f)$ : 
$\pi_{0}(X) \rightarrow \pi_{0}(Y)$. Заметим, что $X$ связно тогда и только тогда, когда связна его геометрическая реализация $|X|$. В частности, справедливо равенство $\pi_{0}(X)=\pi_{0}(|X|)$. Поэтому для любой слабой эквивалентности $f: X \rightarrow Y$ в $S p$ отображение $\pi_{0}(f)$ является биекцией.

(с) При $x, y \in X$ мы говорим, что $x \sim y$, если $x$ и $y$ принадлежат одной и той же компоненте связности пространства $X$. Если $X$ - комплекс Кана, то $x \sim y$ тогда и только тогда, когда существует отображение $\alpha: \Delta[1] \rightarrow X$, такое, что $\alpha(0)=x$ и $\alpha(1)=y($ см. [1, лемма 6.1]).

1.2.9. Лемма. (а) Пусть $f: X \rightarrow Y$ - тривиальное расслоение. Тогда его пространство сечений $\operatorname{Map}_{Y}(Y, X)$ непусто и связно.

(b) Пусть $f: X \rightarrow Y$ - расслоение в $S p$. Тогда $f$ тривиалъно в том и только в том случае, когда комплекс Кана $f^{-1}(y)$ стлгиваем для любого у $\in Y$.

(с) Пусть $f: X \rightarrow Y$ - отображсние расслоений над $Z$ в $S p$. Тогда $f$ является слабой эквивалентностъю в том и только в том случае, когда отображсение слоев $f_{z}: X_{z} \rightarrow Y_{z}$ - слабая эквивалентность для любого $z \in Z$.

Доказательство. (а) Поскольку $Y$ кофибрантно, проекция $X^{Y} \rightarrow Y^{Y}$ является тривиальным расслоением (в силу 1.1.9(a)). Поэтому его слой $\operatorname{Map}_{Y}(Y, X)$ - стягиваемый комплекс Кана (в силу 1.1.7(b)), и потому он непуст и связен в силу $1.2 .8(\mathrm{~b})$.

(b) Согласно последнему утверждению теоремы 1.2.6, расслоение $f$ тривиально тогда и только тогда, когда его пулбэк $\tau^{*}(f)$ - тривиальное расслоение для любого $\tau: \Delta[n] \rightarrow Y$. Таким образом, можно предположить, что $Y=\Delta[n]$. Тогда для любого $y \in \Delta[n]$ включение $y: \Delta[0] \rightarrow \Delta[n]$ является слабой эквивалентностью. Таким образом, $X_{y} \rightarrow X$ - слабая эквивалентность, потому что категория $S p$ правильна справа. Значит, в силу свойства «2 из $3 » f$ является слабой эквивалентностью тогда и только тогда, когда слабой эквивалентностью является $X_{y} \rightarrow \Delta[0]$.

(с) Это будет доказано в 1.3.13.

\section{3. Симплициальные пространства.}

1.3.1. Обозначения. (а) Под категорией симплициалъных пространств мы понимаем категорию функторов $s S p=\operatorname{Fun}\left(\Delta^{o p}, S p\right)=\operatorname{Fun}\left(\Delta^{o p} \times \Delta^{o p}, S e t\right)$.

(b) Для $X \in s S p$ и $n, m \geqslant 0$ положим $X_{n}:=X([n]) \in S p$ и $X_{n, m}:=\left(X_{n}\right)_{m} \in$ Set. Для любого морфизма $f: X \rightarrow Y$ в $s S p$ обозначим через $f_{n}: X_{n} \rightarrow Y_{n}$ соответствующий морфизм в $S p$.

(с) Для любого $\tau:[n] \rightarrow[m]$ в $\Delta$ обозначим через $\tau^{*}: X_{m} \rightarrow X_{n}$ индуцированное отображение пространств. Положим также $\delta_{k_{0}, \ldots, k_{m}}:=\left(\delta^{k_{0}, \ldots, k_{m}}\right)^{*}: X_{n} \rightarrow$ $X_{m}, d_{i}:=\left(d^{i}\right)^{*}: X_{n} \rightarrow X_{n-1}$ и $e_{i}:=\left(e^{i}\right)^{*}: X_{n} \rightarrow X_{m}$.

(d) Согласно 1.1.2, категория $s S p$ декартова и в ней существуют все пределы и копределы. Для $X, Y \in s S p$ определим пространство отображений $\operatorname{Map}(Y, X):=\left(X^{Y}\right)_{0} \in S p$.

1.3.2. Два вложения $S p \hookrightarrow s S p$. (а) Обозначим через $\operatorname{diag}: S p \rightarrow s S p$ (соответственно $\operatorname{diag}:$ Set $\rightarrow S p$ ) отображение, сопоставляющее каждому объекту $X$ постоянное симплициальное пространство (соответственно множество) $[n] \mapsto X, \tau \mapsto \mathrm{Id}_{X}$. Для любого $X \in S p$, обозначим постоянное симплициальное пространство $\operatorname{diag}(X) \in s S p$ просто через $X$. 
(b) Вложение $\operatorname{diag}: \operatorname{Set} \rightarrow S p$ индуцирует вложение $\operatorname{disc:} \operatorname{Sp}=\operatorname{Fun}\left(\Delta^{o p}, \operatorname{Set}\right)$ $\rightarrow s S p=\operatorname{Fun}\left(\Delta^{o p}, S p\right)$. Тогда образ вложения disc состоит из дискретных симплициальных пространств, а именно из $X \in s S p$, таких, что $X_{n} \in S p$ дискретно (т. е. каждое отображение $\Delta[1] \rightarrow X_{n}$ постоянно) для всех $n$.

(c) Положим $F[n]:=\operatorname{disc}(\Delta[n]), \partial F[n]:=\operatorname{disc}(\partial \Delta[n])$ и $F^{i}[n]:=\operatorname{disc}\left(\Lambda^{i}[n]\right)$.

1.3.3. Стандартный бисимплекс. (а) Для $n, m \geqslant 0$ положим $[n, m]:=$ $([n],[m]) \in \Delta^{2}$ и $\square[n, m]:=F[n] \times \Delta[m] \in s S p$. В частности, $F[n]=\square[n, 0]$, $\Delta[m]=\square[0, m]$ и $\mathrm{pt}=F[0]=\Delta[0]$.

(b) Заметим, что $\square[n, m]-$ не что иное, как функтор $\operatorname{Hom}_{\Delta \times \Delta}(\cdot,[n, m])$. Тогда в силу леммы Йонеды получаем отождествления $\operatorname{Hom}\left(\square[n, m], \square\left[n^{\prime}, m^{\prime}\right]\right)=$ $\operatorname{Hom}\left([n, m],\left[n^{\prime}, m^{\prime}\right]\right)$ и $\operatorname{Hom}(\square[n, m], X)=X_{n, m}$. В частности, справедливы отождествления $\operatorname{Map}(F[n], X)=X_{n}$ и $\operatorname{Hom}(F[n], F[m])=\operatorname{Hom}([n],[m])$.

(c) Положим также $\partial \square[n, m]:=(\partial F[n] \times \Delta[m]) \sqcup_{(\partial F[n] \times \partial \Delta[m])}(F[n] \times \partial \Delta[m])$ и $X_{\partial n}:=\operatorname{Map}(\partial F[n], X)$.

1.3.4. Слои. (а) Для $X \in s S p$ пишем $x \in X$ вместо $x \in X_{0,0}$ и $x \sim y \in X$ вместо $x \sim y \in X_{0}$. Согласно 1.3.3(b), каждому $x \in X$ соответствует отображение $x:$ pt $\rightarrow X$.

(b) Как и в 1.2.4, для любого морфизма $f: Y \rightarrow X$ в $s S p$ обозначим через $f^{-1}(x)$ или $Y_{x}$ расслоенное произведение $\{x\} \times_{X} Y:=\mathrm{pt} \times_{x, X} Y$ и назовем его слоем морфизма $f$ в точке $x$.

(с) Для любых $Z \in s S p$ и $X, Y \in s S p / Z$ обозначим через $\mathscr{M} a p_{Z}(X, Y) \in s S p$ слой морфизма $Y^{X} \rightarrow Z^{X}$ над проекцией $(X \rightarrow Z) \in Z^{X}$. Также обозначим через $\operatorname{Map}_{Z}(X, Y):=\mathscr{M} a p_{Z}(X, Y)_{0} \in S p$ нулевое пространство слоя $\mathscr{M}_{a} p_{Z}(X, Y)$.

1.3.5. Определение. Будем говорить, что отображение $f: X \rightarrow Y$ в $s S p$ является расслоением (Риди), если для любого $n \geqslant 0$ индуцированное отображение $\bar{f}_{n}: X_{n} \rightarrow Y_{n} \times_{Y_{\partial n}} X_{\partial n}$ является расслоением Кана в $S p$.

1.3.6. Теорема. Категория sSp обладает структурой декартовой правильной модельной категории, такой, что расслоения являются расслоениями Риди, а корасслоения и слабые эквивалентности определяются покоординатно, m.е. $f: X \rightarrow Y$ является корасслоением (слабой эквивалентностъю), если таково $f_{n}: X_{n} \rightarrow Y_{n}$ для всех $n$.

Доказательство. Все утверждения кроме утверждения, что эта модельная категория декартова, доказаны в [1, гл. IV, теорема 3.9]. Для доказательства оставшегося утверждения используем лемму 1.1.10. Теперь утверждение следует из того факта, что пушауты, произведения, корасслоения и слабые эквивалентности определяются покоординатно, а модельная категория $S p$ декартова.

1.3.7. Замечания. (а) Из леммы 1.1 .3 следует, что отображение $f: X \rightarrow Y$ в $s S p$ является расслоением тогда и только тогда, когда оно поднимаемо справа относительно всех включений $(\partial F[n] \times \Delta[m]) \sqcup_{\left(\partial F[n] \times \Lambda^{i}[m]\right)}\left(F[n] \times \Lambda^{i}[m]\right) \hookrightarrow$ $\square[n, m]$.

(b) Если $f: X \rightarrow Y$ - расслоение Риди, то отображение $f_{n}: X_{n} \rightarrow Y_{n}$ является расслоением для всех $n$. Действительно, $f_{0}=\bar{f}_{0}$ - расслоение по определению, $f^{F[n]}: X^{F[n]} \rightarrow Y^{F[n]}$ - расслоение в силу 1.1.9(а), так что $f_{n}=\left(f^{F[n]}\right)_{0}$ тоже расслоение. 
(c) Пусть $X \rightarrow Y$-расслоение в $s S p$, и пусть $i: A \rightarrow B$-корасслоение над $Y$. Тогда отображение $X^{B} \rightarrow Y^{B} \times_{Y^{A}} X^{A}$ является расслоением по теореме 1.3.6. Взяв нулевые пространства слоев над проекцией $(B \rightarrow Y) \in Y^{B}$, заключаем, что отображение $i^{*}: \operatorname{Map}_{Y}(B, X) \rightarrow \operatorname{Map}_{Y}(A, X)$ является расслоением. В частности, $\operatorname{Map}_{Y}(B, X) \in S p$ - комплекс Кана.

1.3.8. Гомотопическая эквивалентность. Пусть $Z \in s S p$ (соответственно $Z \in S p)$.

(а) Будем говорить, что отображения $f: X \rightarrow Y$ и $g: X \rightarrow Y$ в $s S p / Z$ (соответственно в $S p / Z)$ гомотопны над $Z$, и писать $f \sim Z g$, если $f \sim g$ как элементы из $\operatorname{Map}_{Z}(X, Y)$.

Заметим, что если $Y \rightarrow Z$ - расслоение, то $\operatorname{Map}_{Z}(X, Y) \in S p$ - комплекс Кана (в силу $1.3 .7(\mathrm{c}))$, так что в силу $1.2 .8(\mathrm{c})$ соотношение $f \sim_{Z} g$ означает, что существует отображение $h: X \times \Delta[1] \rightarrow Y$ над $Z$, такое, что $\left.h\right|_{0}:=\left.h\right|_{X \times\{0\}}=f$ и $\left.h\right|_{1}:=\left.h\right|_{X \times\{1\}}=g$.

(b) Будем говорить, что отображение $f: X \rightarrow Y$ - гомотопическая эквивалентность над $Z$, если существует отображение $g: Y \rightarrow X$ над $Z$, называемое гомотопически обратным к $f$, такое, что $f \circ g \sim_{Z} \operatorname{Id}_{Y}$ и $g \circ f \sim_{Z} \operatorname{Id}_{X}$.

1.3.9. Замечания. (а) Пусть $f: X \rightarrow Y$ - гомотопическая эквивалентность над $Z$ с гомотопически обратным $g$. Тогда для любого $\tau: Z^{\prime} \rightarrow Z$ пулбэк $\tau^{*}(f)$ является гомотопической эквивалентностью над $Z^{\prime}$ с гомотопически обратным $\tau^{*}(g)$. Аналогично, для любого $K \in s S p$ отображение $f^{K}: X^{K} \rightarrow Y^{K}$ является гомотопической эквивалентностью с гомотопически обратным $g^{K}: Y^{K} \rightarrow X^{K}$. Кроме того, композиция гомотопических эквивалентностей является гомотопической эквивалентностью.

(b) По определению, если $f \sim_{Z} g$ в $s S p$, то $f_{n} \sim_{Z_{n}} g_{n}$ в $S p$ для всех $n$.

(c) Если $f \sim_{Z} g$, то $f$ является слабой эквивалентностью тогда и только тогда, когда слабой эквивалентностью является $g$. Действительно, утверждение для $S p$ следует из того факта, что если $f \sim_{Z} g$, то геометрические реализации удовлетворяют соотношению $|f| \sim|Z||g|$, а утверждение для $s S p$ следует из утверждения для $S p$ и из (b).

(d) Как и в (c), можно показать, что каждая гомотопическая эквивалентность является слабой эквивалентностью.

1.3.10. Сильный деформационный ретракт. Пусть $Z \in s S p$ и $X \in s S p / Z$ (соответственно $Z \in S p$ и $X \in S p / Z$ ).

(а) Будем говорить, что $Y \subset X-$ сильный деформационный ретракт над $Z$, если существует отображение $h: X \times \Delta[1] \rightarrow X$ над $Z$, такое, что $\left.h\right|_{0}=\operatorname{Id}_{X}$, $\left.h\right|_{1}(X) \subset Y$ и $\left.h\right|_{Y \times \Delta[1]}$ совпадает с $Y \times \Delta[1] \stackrel{\mathrm{pr}_{1}}{\longrightarrow} Y \subset X$.

(b) Если $Y \subset X$ - сильный деформационный ретракт над $Z$, то вложение $i: Y \hookrightarrow X$ является гомотопической эквивалентностью над $Z$, а $\left.h\right|_{1}: X \rightarrow Y-$ ее гомотопически обратный. Таким образом, $i$ - тривиальное корасслоение в силу $1.3 .9(\mathrm{~d})$, а $Y \rightarrow Z$ - ретракт морфизма $X \rightarrow Z$. В частности, $Y \rightarrow Z-$ расслоение, если $X \rightarrow Z-$ расслоение (в силу аксиомы СМ3).

(с) Обратно, если включение $i: Y \hookrightarrow X$-тривиальное корасслоение расслоений над $Z$, то $i(Y) \subset X-$ сильный деформационный ретракт над $Z$.

Доказательство п. (c). Поскольку $i: Y \rightarrow X$ - тривиальное корасслоение, в то время как $Y \rightarrow Z$ - расслоение, существует отображение $p: X \rightarrow Y$ над $Z$, такое, что $p \circ i=\operatorname{Id}_{Y}$. Далее, индуцированное отображение $(X \times \partial \Delta[1]) \sqcup_{(Y \times \partial \Delta[1])}$ 
$(Y \times \Delta[1]) \hookrightarrow X \times \Delta[1]$ является тривиальным корасслоением (по лемме 1.1.10). Поскольку $X \rightarrow Z$ - расслоение, существует отображение $h: X \times \Delta[1] \rightarrow X$ над $Z$, такое, что $\left.h\right|_{0}=\operatorname{Id}_{X},\left.h\right|_{1}=p$ и $\left.h\right|_{Y \times \Delta[1]}=\operatorname{pr}_{1}$.

1.3.11. Лемма. (а) Морфизм $f: X \rightarrow Y$ над $Z$ является гомотопической эквивалентностью над $Z$ тогда и только тогда, когда для любого отображения $\alpha: K \rightarrow Z$ в sSp индуиированное отображение $\pi_{0}\left(\operatorname{Map}_{Z}(K, X)\right) \rightarrow$ $\pi_{0}\left(\operatorname{Map}_{Z}(K, Y)\right)$ является биекиией.

(b) Каждая слабая эквивалентность расслоений является гомотопической эквивалентностью. В частности, пулбэк слабой эквивалентности расслоений есть слабая эквивалентность.

(с) Для любого расслоения $X \rightarrow Y \times \Delta[1]$ существует слабая эквивалентность $\left.\left.X\right|_{0} \rightarrow X\right|_{1}$ расслоений над $Y$.

(d) Пусть $f: Y_{A} \rightarrow A$ - расслоение, и пусть $A \hookrightarrow B$ - тривиальное корасслоение. Тогда существует расслоение $g: Y_{B} \rightarrow B$, сужение которого на $A$ ecms $f$.

Доказательство. (а) Если $f$ - гомотопическая эквивалентность, то индуцированное отображение $\operatorname{Map}_{Z}(K, X) \rightarrow \operatorname{Map}_{Z}(K, Y)$ - также гомотопическая эквивалентность (в силу 1.3.9(a)), так что нужное утверждение следует из $1.3 .9(\mathrm{~d})$ и 1.2.8(b). Обратно, применяя предположение к проекции $Y \rightarrow Z$, находим морфизм $g: Y \rightarrow X$ над $Z$, такой, что $f \circ g \sim_{Z} \operatorname{Id}_{Y}$. Далее, применяя предположение к проекции $X \rightarrow Z$, получаем, что $g \circ f \sim_{Z} \operatorname{Id}_{X}$.

(b) Согласно 1.1.7(c) и 1.3.9(a), достаточно отдельно рассмотреть случаи тривиального корасслоения и тривиального расслоения. Если $f$-тривиальное корасслоение, то утверждение следует из 1.3.10(b),(c). Если $f$-тривиальное расслоение, то утверждение следует из (а). Действительно, каждое отображение $\operatorname{Map}_{Z}(K, X) \rightarrow \operatorname{Map}_{Z}(K, Y)$ является тривиальным расслоением, так что соответствующее отображение для $\pi_{0}$ является биекцией в силу 1.2.8(b). Последнее утверждение следует из 1.3.9(a).

(с) Поскольку каждое отображение $\delta^{i}: \Delta[0] \hookrightarrow \Delta[1]$ является тривиальным корасслоением, индуцированное отображение $\left(\delta^{i}\right)^{*}: X^{\Delta[1]} \rightarrow X \times_{(Y \times \Delta[1])}(Y \times$ $\Delta[1])^{\Delta[1]}$ есть тривиальное расслоение. Взяв пулбэк относительно включения $Y \hookrightarrow(Y \times \Delta[1])^{\Delta[1]}$, соответствующего $\operatorname{Id}_{Y \times \Delta[1]}$, получаем тривиальное расслоение $\widetilde{X}:=X^{\Delta[1]} \times\left._{(\Delta[1] \times Y)^{\Delta[1]}} Y \rightarrow X\right|_{i}$ над $Y$. Таким образом, и $\left.X\right|_{0}$, и $\left.X\right|_{1}$ гомотопически эквивалентны $\widetilde{X}$ над $Y$ (в силу $(\mathrm{b}))$.

(d) Это будет доказано в 3.2.3.

1.3.12. Замечание. Из леммы 1.3.11(b) и замечания 1.2.8(b) следует, что комплекс Кана $X \in S p$ стягиваем тогда и только тогда, когда проекция $X \rightarrow \mathrm{pt}$ является гомотопической эквивалентностью. По определению это происходит тогда и только тогда, когда $X$ непусто и отображение $\mathrm{Id}_{X}$ гомотопно постоянному отображению $X \rightarrow\{x\} \subset X$.

1.3.13. Доказательство леммы 1.2.9(c). Если $f$-слабая эквивалентность, то каждое $f_{z}: X_{z} \rightarrow Y_{z}$ является слабой эквивалентностью по лемме 1.3.11(b).

Обратно, запишем $f$ как $p \circ i$, где $i: X \rightarrow X^{\prime}$ - тривиальное корасслоение, a $p: X^{\prime} \rightarrow Y$ - расслоение. Согласно части «только в том случае», каждое $i_{z}$ есть слабая эквивалентность. Поскольку каждое $f_{z}$-тривиальное расслоение 
по предположению, каждое $p_{z}$ есть слабая эквивалентность по свойству «2 из $3 »$. Поскольку $p_{z}: X_{z}^{\prime} \rightarrow Y_{z}$ - расслоение, оно является тривиальным расслоением. Тогда все слои каждого $p_{z}$ стягиваемы, так что все слои отображения $p$ стягиваемы. Поэтому $p$ - тривиальное расслоение по лемме $1.2 .9(\mathrm{~b})$ и, значит, $f-$ слабая эквивалентность.

1.4. Пространства Сигала. Наше изложение близко следует статье РезKa [6].

1.4.1. Обозначения. (а) Будем говорить, что $X \in s S p$ является пространством Сигала, если $X$ фибрантно и

$$
\varphi_{n}=: \delta_{01} \times_{\delta_{1}} \cdots \times_{\delta_{n-1}} \delta_{n-1, n}: X_{n} \rightarrow X_{1} \times_{X_{0}} \cdots \times_{X_{0}} X_{1}
$$

- слабая эквивалентность для любого $n \geqslant 2$.

(b) Так как модельная категория Риди декартова, то для любого фибрантного объекта $X \in S p$ отображение $\varphi_{n}$ есть расслоение. Таким образом, фибрантный объект $X \in s S p$ является пространством Сигала тогда и только тогда, когда каждое отображение $\varphi_{n}$ есть тривиальное расслоение.

1.4.2. «Объекты» и «пространства отображений». Пусть $X$ - пространство Сигала.

(а) Под его пространством объектов мы понимаем пространство $X_{0}$. Положим $\mathrm{Ob} X:=X_{0,0}$ и назовем его множеством объектов пространства Сигала $X$. Как и в 1.3.4, пишем $x \in X$ вместо $x \in \mathrm{Ob} X$.

(b) Для любых $x, y \in X$ обозначим через $\operatorname{map}(x, y)=\operatorname{map}_{X}(x, y) \in S p$ слой отображения $\left(\delta_{0}, \delta_{1}\right): X_{1} \rightarrow X_{0} \times X_{0}$ над $(x, y)$. Заметим, что поскольку $X$ фибрантно, отображение $\left(\delta_{0}, \delta_{1}\right)$ есть расслоение, так что каждое пространство $\operatorname{map}(x, y)$ является комплексом Кана. Для любых $x, y, z \in X$ обозначим через $\operatorname{map}(x, y, z)$ слой отображения $\left(\delta_{0}, \delta_{1}, \delta_{2}\right): X_{3} \rightarrow\left(X_{0}\right)^{3}$ над $(x, y, z)$.

(c) Для любого $x \in X$ положим $\operatorname{id}_{x}:=\delta_{0,0}(x) \in \operatorname{map}_{X}(x, x)$.

(d) Назовем отображение пространств Сигала $f: X \rightarrow Y$ вполне строгим, если для любых $x, y \in X$ индуцированное отображение $\operatorname{map}_{X}(x, y) \rightarrow \operatorname{map}_{Y}(f(x)$, $f(y))$ есть слабая эквивалентность.

1.4.3. Гомотопическая категория. (а) Пусть $X-$ пространство Сигала, a $x, y, z \in X$. Тогда тривиальное расслоение $\varphi_{2}: X_{2} \rightarrow X_{1} \times_{X_{0}} X_{1}$ индуцирует тривиальное расслоение $\operatorname{map}(x, y, z) \rightarrow \operatorname{map}(x, y) \times \operatorname{map}(y, z)$ (в силу 1.1.7(b)), которое по лемме 1.2.9(a) имеет сечение $s$, единственное с точностью до гомотопии. Таким образом, имеется корректно определенное отображение

$$
[s]:=\pi_{0}(s): \pi_{0}(\operatorname{map}(x, y)) \times \pi_{0}(\operatorname{map}(y, z)) \rightarrow \pi_{0}(\operatorname{map}(x, y, z)) .
$$

(b) Отображение $\delta_{02}: X_{2} \rightarrow X_{1}$ индуцирует отображение $\delta_{02}: \operatorname{map}(x, y, z) \rightarrow$ $\operatorname{map}(x, z)$. Поэтому для любых $[\alpha] \in \pi_{0}(\operatorname{map}(x, y))$ и $[\beta] \in \pi_{0}(\operatorname{map}(y, z))$ можно определить композицию

$$
[\beta] \circ[\alpha]:=\pi_{0}\left(\delta_{02}\right)([s]([\alpha],[\beta])) \in \pi_{0}(\operatorname{map}(x, z)) .
$$

Нетрудно доказать (см. [6, предложение 5.4]), что эта композиция ассоциативна и удовлетворяет соотношению $[\alpha] \circ\left[\mathrm{id}_{x}\right]=[\alpha]=\left[\operatorname{id}_{y}\right] \circ[\alpha]$ для всех $\alpha \in \operatorname{map}(x, y)$.

(c) Используя (b), можно сопоставить пространству Сигала $X$ его гомотопическую категорию Но $X$, объекты которой - это Ob $X$, морфизмы определены равенством $\operatorname{Hom}_{\text {Но } X}(x, y):=\pi_{0}\left(\operatorname{map}_{X}(x, y)\right)$, композиция определена в $(1.2)$, а тождественное отображение есть $\left[\mathrm{id}_{x}\right] \in \operatorname{Hom}_{\mathrm{Ho}} X(x, x)$. 
1.4.4. Полные пространства Сигала. Пусть $X$ - пространство Сигала.

(а) Будем говорить, что $\alpha \in \operatorname{map}_{X}(x, y) \subset X_{1}$ - гомотопическая эквивалентность, если соответствующий морфизм $[\alpha] \in \operatorname{Hom}_{\text {Но } X}(x, y)$ является изоморфизмом. Явным образом это означает, что существует $\beta \in \operatorname{map}(y, x)$, такое, что $[\beta] \circ[\alpha]=\left[\mathrm{id}_{x}\right]$ и $[\alpha] \circ[\beta]=\left[\mathrm{id}_{y}\right]$.

(b) Пусть $X_{h e q} \subset X_{1}$ - максимальное подпространство, такое, что каждое $\alpha \in X_{h e q}$ есть гомотопическая эквивалентность. Нетрудно доказать (см. [6, лемма 5.8]), что $X_{h e q} \subset X_{1}$ - объединение компонент связности.

(c) Заметим, что так как каждый морфизм $\left[\mathrm{id}_{x}\right]$ является изоморфизмом, то $\operatorname{id}_{x} \in X_{h e q}$ для любого $x \in X$. Поэтому отображение $s_{0}:=\delta_{0,0}: X_{0} \rightarrow X_{1}$ пропускается через $X_{h e q}$. Будем говорить, что $X$ - полное пространство Сигала, если отображение $s_{0}: X_{0} \rightarrow X_{h e q}-$ слабая эквивалентность.

1.4.5. Лемма. Пусть $X \in s S p$ - пространство Сигала.

(а) Пусть $X_{1}^{\prime} \subset X_{1}$-объединение компонент связности, пересекающихся $c$ $s_{0}\left(X_{0}\right)$, u nyсть $X_{3}^{\prime}:=\delta_{02}^{-1}\left(X_{1}^{\prime}\right) \cap \delta_{13}^{-1}\left(X_{1}^{\prime}\right) \subset X_{3}$. Тогда $X_{1}^{\prime} \subset X_{\text {heq }} u \delta_{12}\left(X_{3}^{\prime}\right)=$ $X_{h e q}$.

(b) $X$ полно тогда и только тогда, когда $\delta_{0}: X_{\text {hеq }} \hookrightarrow X_{1} \rightarrow X_{0}-$ тривиальное расслоение.

Доказательство. (а) Так как $X_{h e q} \subset X_{1}$ - объединение связных компонент, то из вложения $s_{0}\left(X_{0}\right) \subset X_{h e q}$ следует, что $X_{1}^{\prime} \subset X_{h e q}$. Далее, для любого $\alpha \in X_{3}^{\prime}$ имеем $\delta_{02}(\alpha), \delta_{13}(\alpha) \in X_{1}^{\prime} \subset X_{h e q}$, так что $\left[\delta_{02}(\alpha)\right]=\left[\delta_{12}(\alpha)\right] \circ\left[\delta_{01}(\alpha)\right]$ и $\left[\delta_{13}(\alpha)\right]=\left[\delta_{23}(\alpha)\right] \circ\left[\delta_{12}(\alpha)\right]$ - изоморфизмы в Но $X$. Поэтому $\left[\delta_{12}(\alpha)\right]$ - изоморфизм, так что $\delta_{12}(\alpha) \in X_{h e q}$.

Обратно, пусть $\alpha \in \operatorname{map}(x, y) \subset X_{h e q}$, и пусть $\beta \in \operatorname{map}(y, x)$ удовлетворяет условиям $[\beta] \circ[\alpha]=\left[\mathrm{id}_{x}\right]$ и $[\alpha] \circ[\beta]=\left[\mathrm{id}_{y}\right]$. Поскольку $\varphi_{3}-$ тривиальное расслоение, оно сюръективно. Таким образом, существует $\gamma \in X_{3}$, такое, что $\delta_{01}(\gamma)=\delta_{23}(\gamma)=\beta$ и $\delta_{12}(\gamma)=\alpha$. Тогда по предположению $\delta_{02}(\gamma) \sim \mathrm{id}_{y}$ и $\delta_{13}(\gamma) \sim \operatorname{id}_{x}$, так что $\gamma \in X_{3}^{\prime}$.

(b) Согласно 1.4.4(b), композиция $X_{h e q} \hookrightarrow X_{1} \rightarrow X_{0} \times X_{0}$ является расслоением, так что проекция $\delta_{0}: X_{h e q} \rightarrow X_{0}$ - также расслоение. Поскольку $\delta_{0} \circ s_{0}=\operatorname{Id}_{X_{0}}$, заключаем, что $s_{0}: X_{0} \rightarrow X_{h e q}$ является слабой эквивалентностью тогда и только тогда, когда $\delta_{0}: X_{h e q} \rightarrow X_{0}$ - тривиальное расслоение.

1.4.6. Декартова структура. Резк показал (см. [6, следствие 7.3]), что если $X-\left(\right.$ полное) пространство Сигала, то $X^{K}$ - также (полное) пространство Сигала для любого $K \in s S p$.

\section{§2. Лемма Йонеды}

\section{1. Левые расслоения.}

2.1.1. Определение. Назовем расслоение $f: X \rightarrow Y$ в $s S p$ левым расслоением, если отображение $\left(f_{*},\left(\delta^{0}\right)^{*}\right): X^{F[1]} \rightarrow X \times_{Y} Y^{F[1]}$, индуцированное отображением $\delta^{0}: F[0] \hookrightarrow F[1]$, является тривиальным расслоением.

2.1.2. Лемма. (а) Пулбэк левого расслоения есть левое расслоение.

(b) Если $f: X \rightarrow Y$ - левое расслоение, mо $f^{Z}: X^{Z} \rightarrow Y^{Z}$ - левое расслоение для любого $Z \in s S p$.

Доказательство. Утверждение (а) следует из того факта, что пулбэк (тривиального) расслоения есть (тривиальное) расслоение (см. 1.1.7(b)). 
(b) По определению отображение $X^{F[1]} \rightarrow X \times_{Y} Y^{F[1]}$ есть тривиальное расслоение. Поскольку модельная структура Риди декартова, заключаем, что $f^{Z}-$ расслоение, в то время как отображение $\left(X^{Z}\right)^{F[1]}=\left(X^{F[1]}\right)^{Z} \rightarrow\left(X \times_{Y} Y^{F[1]}\right)^{Z}=$ $X^{Z} \times_{Y^{Z}}\left(Y^{Z}\right)^{F[1]}$ есть тривиальное расслоение (мы использовали 1.1.9). Таким образом, $f^{Z}: X^{Z} \rightarrow Y^{Z}-$ левое расслоение.

2.1.3. Лемма. Пусть $f: X \rightarrow Y$ - расслоение в sSp. Следующие условия эквивалентны:

(a) $f$-левое расслоение,

(b) для любого $n \geqslant 1$ отображсение $\left(f_{*},\left(\delta^{0}\right)^{*}\right): X^{F[n]} \rightarrow X \times_{Y} Y^{F[n]}$, индуиированное отображсением $\delta^{0}:[0] \hookrightarrow[n]$, есть тривиальное расслоение,

(с) для любого $n \geqslant 1$ отображсение $p_{n}: X_{n} \rightarrow X_{0} \times_{Y_{0}} Y_{n}$, индуцированное отображсением $\delta^{0}:[0] \hookrightarrow[n]$, есть тривиальное расслоение.

Доказательство. $(\mathrm{a}) \Longrightarrow(\mathrm{b})$. Согласно (a) и 1.1.9(b), отображение

$$
p: X^{F[1] \times F[n]}=\left(X^{F[1]}\right)^{F[n]} \rightarrow\left(X \times_{Y} Y^{F[1]}\right)^{F[n]}=X^{F[n]} \times_{Y^{F[n]}} Y^{F[1] \times F[n]}
$$

является тривиальным расслоением. Поскольку тривиальные расслоения устойчивы при переходе к ретракту (аксиома СМ3), остается показать, что отображение $X^{F[n+1]} \rightarrow X \times_{Y} Y^{F[n+1]}$ - ретракт отображения $p$. Достаточно показать, что $\delta^{0}:[0] \hookrightarrow[n+1]$ - ретракт отображения $\delta^{0}:[n] \times[0] \hookrightarrow[n] \times[1]$. Рассмотрим отображения $[n+1] \stackrel{\alpha}{\longrightarrow}[n] \times[1] \stackrel{\beta}{\longrightarrow}[n+1]$, где $\alpha(0)=(0,0), \alpha(i)=(i-1,1)$ для $i \geqslant 1$ и $\beta(i, j)=(i+1) j$. Тогда $\beta \circ \alpha=\mathrm{Id}, \alpha(0) \in[n] \times\{0\}$ и $\beta([n] \times\{0\})=0$, так что $\alpha$ и $\beta$ реализуют $\delta^{0}:[0] \hookrightarrow[n+1]$ как ретракт отображения $\delta^{0}:[n] \times[0] \hookrightarrow[n] \times[1]$.

$(\mathrm{b}) \Longrightarrow(\mathrm{c})$. Достаточно перейти к нулевым пространствам.

$(\mathrm{c}) \Longrightarrow(\mathrm{a})$. Сначала предположим, что $Y$ фибрантно. Так как $f$ - расслоение, то $X$ фибрантно и индуцированное отображение $X^{F[1]} \rightarrow X \times_{Y} Y^{F[1]}$ является расслоением. Остается показать, что каждое отображение $\left(X^{F[1]}\right)_{n} \rightarrow$ $X_{n} \times_{Y_{n}}\left(Y^{F[1]}\right)_{n}$ - слабая эквивалентность. Так как отображение $X_{n} \rightarrow X_{0} \times Y_{0} Y_{n}$ есть тривиальное расслоение, то его пулбэк

$$
X_{n} \times_{Y_{n}}\left(Y^{F[1]}\right)_{n} \rightarrow\left(X_{0} \times_{Y_{0}} Y_{n}\right) \times_{Y_{n}}\left(Y^{F[1]}\right)_{n}=X_{0} \times_{Y_{0}}\left(Y^{F[1]}\right)_{n}
$$

- тривиальное расслоение. Остается показать, что отображение $\left(X^{F[1]}\right)_{n} \rightarrow$ $X_{0} \times_{Y_{0}}\left(Y^{F[1]}\right)_{n}$, или, что эквивалентно, $q_{n}:\left(X^{F[1] \times F[n]}\right)_{0} \rightarrow X_{0} \times_{Y_{0}}\left(Y^{F[1] \times F[n]}\right)_{0}$, есть слабая эквивалентность.

Мы рассуждаем, как в [6, лемма 10.3]. Пусть $\gamma^{i}:[n+1] \rightarrow[n] \times[1]$ (соответственно $\left.\epsilon^{i}:[n] \rightarrow[n] \times[1]\right)$ - отображение, переводящее $j$ в $(j, 0)$, если $j \leqslant i$, и в $(j-1,1)$ (соответственно в $(j, 1))$ в противном случае. Тогда отображения $\gamma^{i}$ и $\epsilon^{i}$ индуцируют для $F[n] \times F[1]$ разложение $F[n+1] \sqcup_{F[n]} \cdots \sqcup_{F[n]} F[n+1]$, где все отображения $F[n] \rightarrow F[n+1]$ являются корасслоениями.

Поэтому получаем для $\left(X^{F[n] \times F[1]}\right)_{0}$ и $X_{0} \times_{Y_{0}}\left(Y^{F[1] \times F[n]}\right)_{0}$ разложения

$$
\left(X^{F[n+1]}\right)_{0} \times\left(X^{F[n]}\right)_{0} \cdots \times\left(X^{F[n]}\right)_{0}\left(X^{F[n+1]}\right)_{0},
$$

$$
\left(X_{0} \times_{Y_{0}}\left(Y^{F[n+1]}\right)_{0}\right) \times_{\left(X_{0} \times_{Y_{0}}\left(Y^{F[n]}\right)_{0}\right)} \cdots \times_{\left(X_{0} \times_{Y_{0}}\left(Y^{F[n]}\right)_{0}\right)}\left(X_{0} \times_{Y_{0}}\left(Y^{F[n+1]}\right)_{0}\right) .
$$

Поскольку $X$ и $Y$ фибрантны, все отображения в обоих расслоенных произведениях являются расслоениями.

Таким образом, отображение $q_{n}$ можно записать как расслоенное произведение отображений $X_{n+1} \rightarrow X_{0} \times_{Y_{0}} Y_{n+1}$ над $X_{n} \rightarrow X_{0} \times_{Y_{0}} Y_{n}$. Поскольку эти 
отображения являются слабыми эквивалентностями в силу (b), заключаем, что $p$ - слабая эквивалентность в силу следствия 3.1.5 (см. далее).

Для общего $Y$ выберем его фибрантную замену $Y^{\prime}$. Тогда по лемме 1.3.11(d) существует расслоение $f^{\prime}: X^{\prime} \rightarrow Y^{\prime}$, сужение которого на $Y$ есть $f$. Мы утверждаем, что $f^{\prime}$ удовлетворяет предположению (с). Поскольку $f^{\prime}$ - расслоение, каждое $p_{n}^{\prime}: X_{n}^{\prime} \rightarrow X_{0}^{\prime} \times_{Y_{0}^{\prime}} Y_{n}^{\prime}$ является расслоением. Таким образом, остается показать, что $p_{n}^{\prime}$ - слабая эквивалентность.

Рассмотрим декартову диаграмму

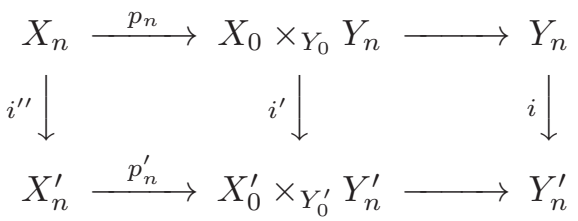

и заметим, что все горизонтальные отображения являются расслоениями. Поскольку $i$ - слабая эквивалентность, а $S p$ правильна справа, заключаем, что $i^{\prime}$ и $i^{\prime \prime}$ - слабые эквивалентности. Поскольку $p_{n}$ - слабая эквивалентность по предположению, $p_{n}^{\prime}$ есть слабая эквивалентность в силу свойства «2 из $3 »$.

Согласно импликации $(\mathrm{c}) \Longrightarrow\left(\right.$ а) для фибрантного $Y$, отображение $q^{\prime}: X^{\prime F[1]}$ $\rightarrow X^{\prime} \times_{Y^{\prime}} Y^{\prime F[1]}$ есть тривиальное расслоение. Поэтому $q$, являясь сужением отображения $q^{\prime}$ на $X \times_{Y} Y^{F[1]}$, также есть тривиальное расслоение.

2.1.4. Замечания. (а) Согласно лемме 2.1.3(c), морфизм $f: X \rightarrow Y$ является левым расслоением тогда и только тогда, когда он поднимаем справа относительно корасслоений

$$
\begin{gathered}
\left(F[n] \times \Lambda^{i}[m]\right) \sqcup_{\left(\partial F[n] \times \Lambda^{i}[m]\right)}(\partial F[n] \times \Delta[m]) \hookrightarrow \square[n, m], \\
(F[n] \times \partial \Delta[m]) \sqcup_{(F[0] \times \partial \Delta[m])}(F[0] \times \Delta[m]) \hookrightarrow \square[n, m] .
\end{gathered}
$$

В частности, морфизм $f: X \rightarrow Y$ есть левое расслоение тогда и только тогда, когда для любого морфизма $\tau: \square[n, m] \rightarrow Y$ пулбэк $\tau^{*}(f): \tau^{*}(X) \rightarrow \square[n, m]$ есть левое расслоение.

(b) Из леммы 2.1.3(c) можно также вывести, что если $f: X \rightarrow Y$ - левое расслоение и $Y$ - (полное) пространство Сигала, то $X$-также (полное) пространство Сигала. В данной работе мы не пользуемся этим фактом.

2.1.5. Лемма. Морфизм $f: X \rightarrow Y$ левых расслоений над $Z$ является слабой эквивалентностью тогда и только тогда, когда отображение слоев $f_{z}:\left(X_{z}\right)_{0} \rightarrow\left(Y_{z}\right)_{0}$ есть слабая эквивалентность для любого $z \in Z$.

Доказательство. Заметим, что $f_{0}: X_{0} \rightarrow Y_{0}-$ морфизм расслоений над $Z_{0}$. Таким образом, $f_{0}$ есть слабая эквивалентность тогда и только тогда, когда индуцированное отображение слоев $f_{z}:\left(X_{z}\right)_{0} \rightarrow\left(Y_{z}\right)_{0}$ есть слабая эквивалентность для всех $z \in Z_{0}$ (по лемме 1.2.9(c)). Таким образом, остается показать, что если $f_{0}$ - слабая эквивалентность, то $f_{n}-$ слабая эквивалентность для любого $n$. Имеется коммутативная диаграмма

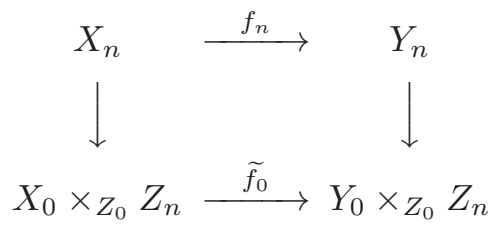


в которой вертикальные отображения являются тривиальными расслоениями по лемме 2.1.3(c). Поскольку $f_{0}$ - слабая эквивалентность, в то время как $X_{0} \rightarrow$ $Z_{0}$ и $Y_{0} \rightarrow Z_{0}$ - расслоения, отображение $\tilde{f}_{0}$ есть слабая эквивалентность в силу следствия 3.1.5. Поэтому $f_{n}$-слабая эквивалентность в силу свойства «2 из $3 »$.

2.1.6. Обозначения. (а) Для $X \in s S p$ и $x \in X$ положим $x \backslash X:=\{x\} \times_{X}$ $X^{F[1]} \rightarrow X$, где отображение $X^{F[1]} \rightarrow X$ индуцировано отображением $\delta^{0}: F[0] \rightarrow$ $F[1]$, и определим $\operatorname{id}_{x}:=s_{0}(x) \in\{x\} \times_{X_{0}} X_{1}=(x \backslash X)_{0}$.

(b) Мы утверждаем, что у проекции $\mathrm{pr}_{2}$ : $\operatorname{id}_{x} \backslash(x \backslash X) \rightarrow x \backslash X$ есть сечение $r$, такое, что $r\left(\mathrm{id}_{x}\right)=\operatorname{id}_{\mathrm{id}_{x}}$. Действительно, положим $A:=(F[1] \times\{0\}) \cup(\{0\} \times$ $F[1]) \subset F[1] \times F[1]$. Тогда отображение $m:[1] \times[1] \rightarrow[1]$, определенное соотношением $m(i, j):=i j$, индуцирует отображение $m: F[1] \times F[1] \rightarrow F[1]$, такое, что $m(A)=0$. Поскольку $\mathrm{id}_{x} \backslash(x \backslash X) \subset X^{F[1] \times F[1]}$ можно записать в виде $\{x\} \times_{X^{A}} X^{F[1] \times F[1]}$, отображение $m^{*}: X^{F[1]} \rightarrow X^{F[1] \times F[1]}$ индуцирует отображение $r: x \backslash X \rightarrow \operatorname{id}_{x} \backslash(x \backslash X)$, которое удовлетворяет соотношениям $r\left(\mathrm{id}_{x}\right)=\operatorname{id}_{\mathrm{id}_{x}}$ и $\mathrm{pr}_{2}$ or $=\mathrm{Id}$.

Следующий результат является одним из основных шагов в доказательстве леммы Йонеды.

2.1.7. Предложение. Для любого левого расслоения $\pi: E \rightarrow X$ и любого $x \in X$ отображсение $\operatorname{ev}_{\mathrm{id}_{x}}: \operatorname{Map}_{X}(x \backslash X, E) \rightarrow \operatorname{Map}_{X}\left(\left\{\operatorname{id}_{x}\right\}, E\right)=\left(E_{x}\right)_{0}$, индуцированное включением $\left\{\mathrm{id}_{x}\right\} \hookrightarrow x \backslash X$, есть тривиальное расслоение.

Доказательство. Поскольку $\left\{\operatorname{id}_{x}\right\} \hookrightarrow x \backslash X$ - корасслоение, в то время как

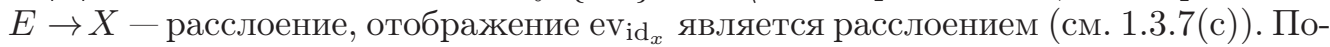
этому остается показать, что для любого $\alpha \in E_{x}$ комплекс Кана $\operatorname{Map}_{X}(x \backslash X, E)_{\alpha}$ $:=\mathrm{ev}_{\mathrm{id}_{x}}^{-1}(\alpha)$ стягиваем (по лемме 1.2.9(b)). Используя замечание 1.3 .12 , достаточно показать, что тождественное отображение комплекса $\operatorname{Map}_{X}(x \backslash X, E)_{\alpha}$ пропускается через стягиваемый комплекс Кана.

Поскольку $E \rightarrow X$ - левое расслоение, проекция $E^{F[1]} \rightarrow E \times_{X} X^{F[1]}$ это тривиальное расслоение. Таким образом, $\alpha \backslash E \rightarrow x \backslash X$, будучи его слоем над $\alpha \in E$, является тривиальным расслоением. Поэтому отображение $\mathrm{ev}_{\mathrm{id}_{x}}^{\prime}$ : $\operatorname{Map}_{x \backslash X}(x \backslash X, \alpha \backslash E) \rightarrow\left((\alpha \backslash E)_{\text {id }_{x}}\right)_{0}$ является расслоением стягиваемых комплексов Кана. Значит, $\mathrm{ev}_{\mathrm{id}_{x}}^{\prime}$ - слабая эквивалентность и, следовательно, тривиальное расслоение. Отсюда вытекает, что его слой $\operatorname{ev}_{\mathrm{id}_{x}}^{\prime-1}\left(\mathrm{id}_{\alpha}\right)=\operatorname{Map}_{x \backslash X}(x \backslash X, \alpha \backslash E)_{\operatorname{id}_{\alpha}}$ есть стягиваемый комплекс Кана.

Заметим, что проекция $\mathrm{pr}_{2}: \alpha \backslash E \rightarrow E$ индуцирует проекцию

$$
\rho: \operatorname{Map}_{x \backslash X}(x \backslash X, \alpha \backslash E)_{\operatorname{id}_{\alpha}} \rightarrow \operatorname{Map}_{X}(x \backslash X, E)_{\alpha} .
$$

Таким образом, достаточно показать, что у $\rho$ есть сечение.

Естественный морфизм $\operatorname{Map}_{X}(x \backslash X, E) \rightarrow \operatorname{Map}_{X^{F[1]}}\left((x \backslash X)^{F[1]}, E^{F[1]}\right)$ индуцирует морфизм $s^{\prime}: \operatorname{Map}_{X}(x \backslash X, E)_{\alpha} \rightarrow \operatorname{Map}_{x \backslash X}\left(\operatorname{id}_{x} \backslash(x \backslash X), \alpha \backslash E\right)$. Согласно 2.1.6, у проекции $\mathrm{pr}_{2}: \operatorname{id}_{x} \backslash(x \backslash X) \rightarrow x \backslash X$ есть сечение $r$, такое, что $r\left(\mathrm{id}_{x}\right)=\mathrm{id}_{\mathrm{id}_{x}}$. Тогда образ морфизма

$$
r^{*} \circ s^{\prime}: \operatorname{Map}_{X}(x \backslash X, E)_{\alpha} \rightarrow \operatorname{Map}_{x \backslash X}\left(\operatorname{id}_{x} \backslash(x \backslash X), \alpha \backslash E\right) \rightarrow \operatorname{Map}_{x \backslash X}(x \backslash X, \alpha \backslash E)
$$

лежит в $\operatorname{Map}_{x \backslash X}(x \backslash X, \alpha \backslash E)_{\operatorname{id}_{\alpha}}$ и $r^{*} \circ s^{\prime}$ является сечением проекции $\rho$. 
2.1.8. Следствие. Пусть $\pi: E \rightarrow X$-левое расслоение, $x \in X, a f$ u отображсения $x \backslash X \rightarrow E$ над $X$, такие, что $f\left(\operatorname{id}_{x}\right) \sim g\left(\operatorname{id}_{x}\right) \in E_{x}$. Тогда $f \sim_{X} g$. $B$ частности, $f$ есть слабая эквивалентность тогда и только тогда, когда $g$ - слабая эквивалентность.

Доказательство. Поскольку $\mathrm{ev}_{\mathrm{id}}$ - тривиальное расслоение (в силу предложения 2.1.7), индуцированное отображение $\pi_{0}\left(\mathrm{ev}_{\mathrm{id}}\right)$ является биекцией в силу 1.2.8(b). Последнее утверждение следует из 1.3.9(c).

2.1.9. Замечания. Пусть $X$ - пространство Сигала. (а) По определению $\left(\delta_{01}, \delta_{12}\right): X_{2} \rightarrow X_{1} \times_{X_{0}} X_{1}$ и его пулбэк $\delta_{12}: X_{0} \times_{s_{0}, X_{1}, \delta_{01}} X_{2} \rightarrow X_{1}$ являются тривиальными расслоениями.

(b) Отображение $\delta_{02}: X_{0} \times_{s_{0}, X_{1}, \delta_{01}} X_{2} \rightarrow X_{1}$ является расслоением. Действительно, $\delta_{12}$ - пулбэк отображения $\left(\delta_{01}, \delta_{02}\right): X_{2} \rightarrow X_{1} \times_{X_{0}} X_{1}$, индуцированный включением $\delta^{01} F[1] \cup \delta^{02} F[1] \hookrightarrow F[2]$. Таким образом, $\delta_{02}-$ расслоение, потому что $X$ фибрантно.

(c) Отображение $\delta_{02}$ из п. (b) - слабая эквивалентность. Действительно, отображение $r=\left(\delta_{0}, \delta_{001}\right): X_{1} \rightarrow X_{0} \times_{s_{0}, X_{1}, \delta_{01}} X_{2}$ удовлетворяет соотношению $\delta_{12} \circ r=\delta_{02} \circ r=\mathrm{Id}$. Поскольку $\delta_{12}$ - слабая эквивалентность (в силу (а)), мы приходим к выводу, что $r$ и $\delta_{02}$ являются слабыми эквивалентностями в силу свойства «2 из $3 »$.

2.1.10. Лемма. Пусть $X$ - пространство Сигала $u x \in X$. Тогда $\mathrm{pr}_{2}$ : $x \backslash X \rightarrow X-$ левое расслоение.

Доказательство. Поскольку $X$ фибрантно, проекция $X^{F[1]} \rightarrow X^{\partial F[1]}=$ $X \times X$ является расслоением, так что ее пулбэк $\operatorname{pr}_{2}: x \backslash X \rightarrow X$ - также расслоение. Остается показать, что отображение $(x \backslash X)^{F[1]} \rightarrow(x \backslash X) \times_{X} X^{F[1]}-$ слабая эквивалентность, или, эквивалентным образом, что отображение $\left((x \backslash X)^{F[1]}\right)_{n} \rightarrow$ $(x \backslash X)_{n} \times_{X_{n}}\left(X^{F[1]}\right)_{n}$ - слабая эквивалентность для всех $n$.

Используя отождествления $\left(X^{F[m]}\right)_{n}=\operatorname{Map}(F[m] \times F[n], X)=\left(X^{F[n]}\right)_{m}$, мы можем переписать последнее отображение в виде $\left(x \backslash X^{F[n]}\right)_{1} \rightarrow\left(x \backslash X^{F[n]}\right)_{0}$ $\times_{\left(X^{F[n]}\right)_{0}}\left(X^{F[n]}\right)_{1}$. Поскольку $X^{F[n]}$ — также пространство Сигала (см. 1.4.6), можно заменить $X$ на $X^{F[n]}$. Остается показать, что отображение $(x \backslash X)_{1} \rightarrow$ $(x \backslash X)_{0} \times_{X_{0}} X_{1}$ - тривиальное расслоение.

Используя разложение $F[1] \times F[1]=F[2] \sqcup_{F[1]} F[2]$, получаем разложение $\left(X^{F[1]}\right)_{1}=X_{2} \times_{\delta_{02}, X_{1}, \delta_{02}} X_{2}$. Отсюда получаем разложение

$$
(x \backslash X)_{1}=\left(\{x\} \times_{X_{1}, \delta_{01}} X_{2}\right) \times_{(x \backslash X)_{0}}\left(\{x\} \times_{X_{0}, \delta_{0}} X_{2}\right),
$$

которое отождествляет отображение $(x \backslash X)_{1} \rightarrow(x \backslash X)_{0} \times_{X_{0}} X_{1}$ с композицией

$$
(x \backslash X)_{1} \stackrel{f}{\longrightarrow}\{x\} \times_{X_{0}, \delta_{0}} X_{2} \stackrel{g}{\longrightarrow}(x \backslash X)_{0} \times_{X_{0}} X_{1} .
$$

Мы утверждаем, что $f$ и $g$ - тривиальные расслоения. Поскольку $g-$ пулбэк расслоения $\left(\delta_{01}, \delta_{12}\right): X_{2} \rightarrow X_{1} \times_{X_{0}} X_{1}$, в то время как $f$-пулбэк расслоения $\delta_{02}:\{x\} \times_{X_{1}, \delta_{01}} X_{2} \rightarrow(x \backslash X)_{0}$ и, следовательно, пулбэк расслоения $\delta_{02}: X_{0} \times_{s_{0}, X_{1}, \delta_{01}} X_{2} \rightarrow X_{1}$, оба утверждения следуют из 2.1.9.

\section{2. $\infty$-категория пространств.}

2.2.1. Обозначения. (а) Для любого $K \in s S p$ обозначим через $[K]$ категорию «бисимплексов симплициального пространства $K$ » Явным образом, 
множество объектов категории $[K]$ есть несвязное объединение $\bigsqcup_{n, m} K_{n, m}$, и для любых $a \in K_{n, m}$ и $b \in K_{n^{\prime}, m^{\prime}}$ множество морфизмов $\operatorname{Mor}_{[K]}(a, b)$ есть множество таких $\tau \in \operatorname{Mor}_{\Delta \times \Delta}\left(\left[n^{\prime}, m^{\prime}\right],[n, m]\right)$, что $\tau^{*}(a)=b$.

(b) Заметим, что имеется естественный изоморфизм категорий $s S p / K \rightarrow$ $\operatorname{Fun}([K], S e t)$. А именно, каждое отображение $f: X \rightarrow K$ определяет функтор $[K] \rightarrow S e t$, который переводит $a \in K_{n, m}$ в $f_{n, m}^{-1}(a) \subset X_{n, m}$. Обратно, каждый функтор $\phi:[K] \rightarrow$ Set определяет $X_{\phi} \in s S p / K$, где $\left(X_{\phi}\right)_{n, m}:=\bigsqcup_{a \in K_{n, m}} \phi(a)$ с очевидными отображениями перехода.

(с) Каждое отображение $\phi: L \rightarrow K$ в $s S p$ индуцирует функтор $[\phi]:[L] \rightarrow[K]$. Тогда биекция из п. (b) отождествляет $\phi^{*}: s S p / K \rightarrow s S p / L$ с пулбэк-функтором $[\phi]^{*}: \operatorname{Fun}([K]$, Set $) \rightarrow \operatorname{Fun}([L]$, Set $)$.

2.2.2. Универсальные множества. Начиная с этого места, зафиксируем бесконечное множество $\mathscr{U}$, который назовем универсальным множеством.

(a) Пусть Set $_{\mathscr{U}} \subset$ Set - категория подмножеств множества $\mathscr{U}$, и пусть $\operatorname{Set}_{|\mathscr{U}|}$ - категория множеств мощности $\leqslant|\mathscr{U}|$. Тогда категория Set является малой, и естественное вложение Set $_{\boldsymbol{U}} \rightarrow \operatorname{Set}_{|\mathscr{U}|}$ представляет собой эквивалентность категорий.

(b) Положим $s S p_{|\mathscr{U}|}:=\operatorname{Fun}\left(\Delta^{o p} \times \Delta^{o p}, \operatorname{Set}_{|\mathscr{U}|}\right) \subset s S p$ и $s p_{\mathscr{U}}:=\operatorname{Fun}\left(\Delta^{o p} \times\right.$ $\Delta^{o p}$, Set$\left._{\mathscr{U}}\right) \subset s S p$.

(c) Для $K \in s S p$ обозначим через $(s S p / K)_{\mathscr{U}}\left(\right.$ соответственно $\left.(s S p / K)_{|\mathscr{U}|}\right)$ полную подкатегорию в $s S p / K$, состоящую из морфизмов $f: X \rightarrow K$, таких,

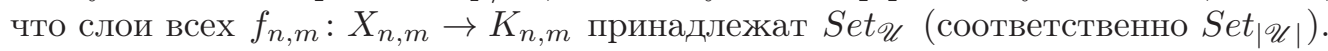

$(\mathrm{d})$ Биекция из 2.2.1(b) индуцирует биекцию между $(s S p / K) \mathscr{U}$ (соответствен-

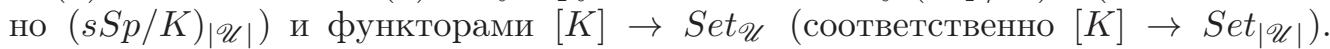
В частности, категория $(s S p / K)_{\mathscr{U}}$ является малой и включение $(s S p / K)_{\mathscr{U}} \rightarrow$ $(s S p / K)|\mathscr{U}|$ является эквивалентностью категорий.

(е) Обозначим через $(L F i b / K)$ ध множество левых расслоений $X \rightarrow K$, принадлежащих $(s S p / K) \mathscr{U}$. Согласно п. (c), 2.2.1(c) и лемме 2.1.2(a), для любого отображения $\phi: L \rightarrow K$ пулбэк-функтор $\phi^{*}: s S p / K \rightarrow s S p / L$ отображает $(L F i b / K) \mathscr{U}$ в $(L F i b / L) \mathscr{U}$.

2.2.3. Основная конструкция. (а) Пусть $\mathfrak{S}_{\mathscr{U}} \in s S p-$ симплициальное пространство, такое, что

- $\left(\mathfrak{S}_{\mathscr{U}}\right)_{n, m}$ есть множество левых расслоений $(L F i b / \square[n, m])_{\mathscr{U}}$;

- для $a \in\left(\mathfrak{S}_{\mathscr{U}}\right)_{n, m}$, соответствующего левому расслоению $p_{a}: E_{a} \rightarrow \square[n, m]$, и $\nu \in \operatorname{Hom}\left(\left[n^{\prime}, m^{\prime}\right],[n, m]\right)=\operatorname{Hom}\left(\square\left[n^{\prime}, m^{\prime}\right], \square[n, m]\right)$ элемент $\nu^{*}(a) \in\left(\mathfrak{S}_{\mathscr{U}}\right)_{n^{\prime}, m^{\prime}}$ соответствует пулбэку $\nu^{*}\left(p_{a}\right): \nu^{*}\left(E_{a}\right) \rightarrow \square\left[n^{\prime}, m^{\prime}\right]$ (мы воспользовались замечанием 2.2.2(e)).

(b) Рассмотрим «универсальное левое расслоение» $p_{\mathscr{U}}: \mathscr{E}_{\mathscr{U}} \rightarrow \mathfrak{S}_{\mathscr{U}}$, где $\left(\mathscr{E} \mathscr{U}_{n, m}\right.$ определено как несвязное объединение $\bigsqcup_{a \in\left(\mathfrak{S}_{\mathscr{U}}\right)_{n, m}}\left(E_{a}\right)_{n, m}$, а $p_{\mathscr{U}}-$ отображение, которое переводит $\left(E_{a}\right)_{n, m}$ в $a \in\left(\mathfrak{S}_{\mathscr{U}}\right)_{n, m}$.

2.2.4. Лемма. Отображение Л $_{\mathscr{U}}: \mathscr{E}_{\mathscr{U}} \rightarrow \mathfrak{S}_{\mathscr{U}}-$ левое расслоение. Для любого $K \in s S p$ отображение $\phi \mapsto \phi^{*}\left(p_{\mathscr{U}}\right)$ определяет биекиию между $\operatorname{Hom}_{s, p}\left(K, \mathfrak{S}_{\mathscr{U}}\right)$ $u(L F i b / K) \mathscr{U}$.

Доказательство. По построению для любого $a \in\left(\mathfrak{S}_{\mathscr{U}}\right)_{n, m}=\operatorname{Hom}(\square[n, m]$, $\left.\mathfrak{S}_{\mathscr{U}}\right)$ пулбэк $a^{*}\left(p_{\mathscr{U}}\right)$ равен $p_{a}: E_{a} \rightarrow \square[n, m]$. В частности, каждый пулбэк 
$a^{*}\left(p_{\mathscr{U}}\right)$ есть левое расслоение. Таким образом, $p_{\mathscr{U}}$ - левое расслоение в силу замечания 2.1.4(a).

Далее, заметим, что для любого $\phi: K \rightarrow \mathfrak{S}_{\mathscr{U}}$ пулбэк $\phi^{*}\left(p_{\mathscr{U}}\right): \phi^{*}\left(\mathscr{E}_{\mathscr{U}}\right) \rightarrow K$ является левым расслоением, удовлетворяющим условию $a^{*}\left(\phi^{*}\left(p_{\mathscr{U}}\right)\right)=$ $(\phi \circ a)^{*}\left(p_{\mathscr{U}}\right) \in(L F i b / \square[n, m])_{\mathscr{U}}$ для любого $a: \square[n, m] \rightarrow K$. Таким образом, $\phi^{*}\left(p_{\mathscr{U}}\right) \in(L F i b / K) \mathscr{U}$.

Обратно, каждое $E \in(L F i b / K)_{\mathscr{U}}$ определяет отображение $\phi_{E}: K \rightarrow \mathfrak{S}_{\mathscr{U}}$, которое переводит $a \in K_{n, m}=\operatorname{Hom}(\square[n, m], K)$ в левое расслоение $a^{*}(E) \rightarrow$ $\square[n, m]$ в $(L F i b / \square[n, m]) \mathscr{U}$. Тогда отображение $E \mapsto \phi_{E}$ обратно к $\phi \mapsto \phi^{*}\left(p_{\mathscr{U}}\right)$.

2.2.5. Замечания. (а) Основной результат этого раздела (теорема 2.2.11) утверждает, что $\mathfrak{S}_{\mathscr{U}}$ - полное пространство Сигала. Это наша модель $\infty$-категории пространств или, более формально, $(\infty, 1)$-категории $(\infty, 0)$-категорий.

(b) Можно показать, что каждое включение $i: \mathscr{U} \hookrightarrow \mathscr{V}$ бесконечных множеств индуцирует вполне строгое отображение $i: \mathfrak{S}_{\mathscr{U}} \hookrightarrow \mathfrak{S}_{\mathscr{V}}$ полных пространств Сигала.

(c) Можно показать (см. [3]), что $\mathfrak{S}_{\mathscr{U}}$ эквивалентно фибрантной замене $N^{f}\left(S p_{\mathscr{U}}, W\right)$ симплициального пространства $N\left(S p_{\mathscr{U}}, W\right)$, которое Резк $[6,3.3]$ сопоставил паре $\left(S p_{\mathscr{U}}, W\right)$, где $W$ обозначает слабые эквивалентности.

(d) Можно также рассмотреть «большую» $(\infty, 1)$-категорию $(\infty, 0)$-категорий $\widehat{\mathfrak{S}}$, таких, что $\widehat{\mathfrak{S}}_{n, m}$ - класс всех левых расслоений $E \rightarrow \square[n, m]$.

(е) В [3] мы обобщаем нашу конструкцию 2.2.3 и строим $(\infty, n+1)$-категорию $(\infty, n)$-категорий.

2.2.6. Обозначения. (а) Для любого $n \geqslant 1$ обозначим через $\mathfrak{S}_{\mathscr{U}}^{(n)} \in s S p$ симплициальное пространство, такое, что $\left(\mathfrak{S}^{(n)}\right)_{m, k}$ есть множество диаграмм $\phi: E^{(0)} \stackrel{\phi_{1}}{\longrightarrow} \cdots \stackrel{\phi_{n}}{\longrightarrow} E^{(n)}$ над $\square[m, k]$, где каждое $E^{(i)} \rightarrow \square[m, k]$ принадлежит $($ LFib/ $\square[m, k]) \mathscr{U}$.

(b) Каждому отображению $\mu:[m] \rightarrow[n]$ сопоставим морфизм $\mu^{*}: \mathfrak{S}_{\mathscr{U}}^{(n)} \rightarrow$ $\mathfrak{S}_{\mathscr{U}}^{(m)}$, который переводит диаграмму $\phi: E^{(0)} \stackrel{\phi_{1}}{\longrightarrow} \cdots \stackrel{\phi_{n}}{\longrightarrow} E^{(n)}$ в диаграмму $\mu^{*}(\phi): E^{(\mu(0))} \rightarrow \cdots \rightarrow E^{(\mu(m))}$, отображения в которой являются композициями отображений $\phi_{i}$.

(c) Пусть $\mathfrak{S}_{\mathscr{U}}^{(w e)} \subset \mathfrak{S}_{\mathscr{U}}^{(1)}-$ симплициальное подпространство, такое, что множество $\left(\mathfrak{S}^{(w e)}\right)_{m, k} \subset\left(\mathfrak{S}^{(1)}\right)_{m, k}$ состоит из слабых эквивалентностей $E^{(0)} \stackrel{\phi}{\longrightarrow}$ $E^{(1)}$ (мы воспользовались леммой 1.3.11(b)).

(d) Имеется естественная проекция $\mathfrak{S}_{\mathscr{U}}^{(n)} \rightarrow\left(\mathfrak{S}_{\mathscr{U}}\right)^{n+1}$, которая сопоставляет диаграмме $\phi$, как в (а), упорядоченный набор $\left(E^{(0)}, \ldots, E^{(n)}\right)$ из $n+1$ элементов. Кроме того, для любого отображения $\mu:[m] \rightarrow[n]$ имеется естественное отображение $\mathfrak{S}_{\mathscr{U}}^{(n)} \stackrel{\mu^{*}}{\longrightarrow} \mathfrak{S}_{\mathscr{U}}^{(m)} \rightarrow\left(\mathfrak{S}_{\mathscr{U}}\right)^{m+1}$.

2.2.7. Замечания. (а) Заметим, что для любых $X, Y \in s S p / K$ задать отобpажение $\phi \in \operatorname{Hom}_{K}(X, Y)$ - это то же самое, что задать отображения $\tau^{*}(\phi) \in$ $\operatorname{Hom}_{\square[n, m]}\left(\tau^{*}(X), \tau^{*}(Y)\right)$ для всех $\phi: \square[n, m] \rightarrow K$, совместимые с композициями. Используя это наблюдение и лемму 2.2.4, заключаем, что для любого $K \in s S p$ имеется естественная биекция между $\operatorname{Hom}_{s S p}\left(K, \mathfrak{S}_{\mathscr{U}}^{(n)}\right)$ и множеством диаграмм $\phi: E^{(0)} \stackrel{\phi_{1}}{\longrightarrow} \cdots \stackrel{\phi_{n}}{\longrightarrow} E^{(n)}$ левых расслоений из $(s S p / K) \mathscr{U}$. 
(b) По определению $\phi \in \operatorname{Hom}_{s S p}\left(K, \mathfrak{S}_{\mathscr{U}}^{(1)}\right)$ принадлежит $\operatorname{Hom}_{s S p}\left(K, \mathfrak{S}_{\mathscr{U}}^{(w e)}\right)$ тогда и только тогда, когда $\phi(a) \in\left(\mathfrak{S}_{\mathscr{U}}^{(w e)}\right)_{n, m}$ для любого $a \in K_{n, m}$. Кроме того, по лемме 2.1.5 это происходит тогда и только тогда, когда $\phi(a) \in\left(\mathfrak{S}_{\mathscr{U}}^{(w e)}\right)_{0,0}$ для любого $a \in K_{0,0}$. Опять используя лемму 2.1.5, видим, что при биекции из п. (а) элементы множества $\operatorname{Hom}_{s S p}\left(K, \mathfrak{S}_{\mathscr{U}}^{(w e)}\right) \subset \operatorname{Hom}_{s S p}\left(K, \mathfrak{S}_{\mathscr{U}}^{(1)}\right)$ соответствуют слабым эквивалентностям $\phi: E^{(0)} \rightarrow E^{(1)}$.

Следующие два предложения и следствие будут доказаны в $\S 4$.

Имеются естественные проекции $\left(\mathfrak{S}_{\mathscr{U}}\right)^{F[n]} \rightarrow\left(\mathfrak{S}_{\mathscr{U}}\right)^{n+1}$ и $\left(\mathfrak{S}_{\mathscr{U}}\right)^{\Delta[1]} \rightarrow\left(\mathfrak{S}_{\mathscr{U}}\right)^{2}$, $i$-я компонента которых есть $\left(\delta^{i}\right)^{*}$.

2.2.8. Предложение. (а) Симплициальное пространство $\mathfrak{S}_{\mathscr{U}} \in s S p$ Puдuфибрантно.

(b) Eстественные проекиии $\mathfrak{S}_{\mathscr{U}}^{(n)} \rightarrow\left(\mathfrak{S}_{\mathscr{U}}\right)^{n+1} u \mathfrak{S}_{\mathscr{U}}^{(w e)} \rightarrow\left(\mathfrak{S}_{\mathscr{U}}\right)^{2}$ являются расслоениями.

(с) Обе композищии $\mathfrak{S}_{\mathscr{U}}^{(w e)} \rightarrow\left(\mathfrak{S}_{\mathscr{U}}\right)^{2} \stackrel{p_{i}}{\longrightarrow} \mathfrak{S}_{\mathscr{U}}$ являются тривиальными расслоениями.

(d) $\left(\mathfrak{S}_{\mathscr{U}}^{(w e)}\right)_{0} \subset\left(\mathfrak{S}_{\mathscr{U}}^{(1)}\right)_{0}$ является объединением компонент связности.

2.2.9. Предложение. (а) Существует гомотопическая эквивалентность $\left(\mathfrak{S}_{\mathscr{U}}\right)^{\Delta[1]} \rightarrow \mathfrak{S}_{\mathscr{U}}^{(w e)}$ над $\left(\mathfrak{S}_{\mathscr{U}}\right)^{2}$.

(b) Для любого $n \in \mathbb{N}$ существует «естественная» гомотопическая эквивалентность $\psi_{\mathscr{U}}^{(n)}: \mathfrak{S}_{\mathscr{U}}^{(n)} \rightarrow\left(\mathfrak{S}_{\mathscr{U}}\right)^{F[n]}$ над $\left(\mathfrak{S}_{\mathscr{U}}\right)^{n+1}$, определенная единственным образом с точностью до гомотопии.

(с) Кроме того, для любого отображения $\mu:[m] \rightarrow[n]$ диаграмма

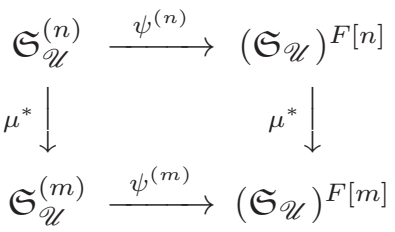

гомотопически коммутативна над $\left(\mathfrak{S}_{\mathscr{U}}\right)^{m+1}, m \cdot e \cdot \mu^{*} \circ \psi^{(n)} \sim_{\left(\mathfrak{S}_{\mathscr{U}}\right)^{m+1}} \psi^{(m)} \circ \mu^{*}$, где отображение $\mathfrak{S}_{\mathscr{U}}^{(n)} \rightarrow\left(\mathfrak{S}_{\mathscr{U}}\right)^{m+1}$ определено в $2.2 .6(\mathrm{~d})$.

2.2.10. Следствие. Пусть $K \in s S p, \alpha, \beta \in \operatorname{Hom}\left(K, \mathfrak{S}_{\mathscr{U}}\right)$, u nycms $E_{\alpha} \rightarrow K$ $u E_{\beta} \rightarrow K$ - cоответствующие левые расслоения. Тогда $\alpha \sim \beta$ в $\left(\mathfrak{S}_{\mathscr{U}}\right)^{K}$ в том и только в том случае, когда левые расслоения $E_{\alpha}$ и $E_{\beta}$ гомотопически эквивалентны над $K$.

Теперь мы можем доказать один из основных результатов статьи.

2.2.11. Теорема. $\mathfrak{S}_{\mathscr{U}}$ - полное пространство Сигала.

Доказательство. Будем обозначать $\mathfrak{S}_{\mathscr{U}}$ просто через $\mathfrak{S}$. Тогда $\mathfrak{S}$ фибрантно в силу предложения 2.2.8(a).

Чтобы показать, что $\mathfrak{S}$ - пространство Сигала, нам нужно доказать, что для любого $n \geqslant 2$ морфизм $\varphi_{n}: \mathfrak{S}_{n} \rightarrow \mathfrak{S}_{1} \times \mathfrak{S}_{0} \cdots \times \mathfrak{S}_{0} \mathfrak{S}_{1}$ есть слабая эквивалентность. Применяя предложение $2.2 .9($ с $)$ к отображениям $\delta^{01}, \ldots, \delta^{n-1, n}:[1] \rightarrow[n]$, 
получаем гомотопически коммутативную диаграмму

$$
\begin{array}{ccc}
\mathfrak{S}^{(n)} & \stackrel{\psi^{(n)}}{\longrightarrow} & \mathfrak{S}^{F[n]} \\
\downarrow & \downarrow \\
\mathfrak{S}^{(1)} \times{ }_{\mathfrak{S}} \cdots \times \times_{\mathfrak{S}} \mathfrak{S}^{(1)} \stackrel{\psi^{(1)} \times \cdots \times \psi^{(1)}}{\longrightarrow} \mathfrak{S}^{F[1]} \times{ }_{\mathfrak{S}} \cdots \times_{\mathfrak{S}} \mathfrak{S}^{F[1]}
\end{array}
$$

Мы хотим показать, что правая вертикальная стрелка-слабая эквивалентность, откуда условия Сигала получаются переходом к нулевым пространствам. Верхняя горизонтальная стрелка - слабая эквивалентность в силу предложения 2.2.9(b). Нижняя горизонтальная стрелка - эквивалентность в силу предложения 2.2.9(b) вместе с наблюдением, что $\mathfrak{S}^{(1)} \rightarrow \mathfrak{S}$ и $\mathfrak{S}^{F[1]} \rightarrow \mathfrak{S}$ являются расслоениями (мы воспользовались предложением 2.2.8 и следствием 3.1.5). Далее, поскольку левая вертикальная стрелка является биекцией, в то время как диаграмма (2.3) гомотопически коммутативна, правая вертикальная стрелка слабая эквивалентность в силу свойства «2 из $3 »$.

Чтобы показать, что $\mathfrak{S}$ полно, нужно доказать, что $\delta_{0}: \mathfrak{S}_{h e q} \rightarrow \mathfrak{S}_{0}-$ тривиальное расслоение (в силу леммы $1.4 .5(\mathrm{~b}))$. Поскольку $p_{0}: \mathfrak{S}^{(w e)} \rightarrow \mathfrak{S}-$ тривиальное расслоение в силу предложения 2.2.8(c), достаточно показать, что отображение $\psi:=\left(\psi^{(1)}\right)_{0}:\left(\mathfrak{S}^{(1)}\right)_{0} \rightarrow\left(\mathfrak{S}^{F[1]}\right)_{0}=\mathfrak{S}_{1}$ из предложения 2.2.9(b) индуцирует эквивалентность $\left(\mathfrak{S}^{(w e)}\right)_{0} \rightarrow \mathfrak{S}_{h e q}$.

$\operatorname{KaK}\left(\mathfrak{S}^{(w e)}\right)_{0} \subset\left(\mathfrak{S}^{(1)}\right)_{0}$, так и $\mathfrak{S}_{h e q} \subset \mathfrak{S}_{1}$ являются объединениями компонент связности (в силу предложения 2.2.8(d) и п. 1.4.4(b)). Поскольку $\psi$ - слабая эквивалентность, остается показать, что $\pi_{0}(\psi)$ индуцирует биекцию $\pi_{0}\left(\left(\mathfrak{S}^{(w e)}\right)_{0}\right)$ $\rightarrow \pi_{0}\left(\mathfrak{S}_{h e q}\right)$.

Согласно предложению 2.2.9(с), имеется следующая гомотопически коммутативная диаграмма:

$$
\begin{aligned}
& \left(\mathfrak{S}^{(1)}\right)_{0} \stackrel{\delta_{12}}{\longleftarrow}\left(\mathfrak{S}^{(3)}\right)_{0} \stackrel{\delta_{02}, \delta_{13}}{\longrightarrow}\left(\mathfrak{S}^{(1)} \times \mathfrak{S}^{(1)}\right)_{0} \stackrel{s_{0} \times s_{0}}{\longleftarrow} \mathfrak{S}_{0} \times \mathfrak{S}_{0} \\
& \psi^{(1)} \downarrow \quad \psi^{(3)} \downarrow \quad \psi^{(1)} \times \psi^{(1)} \downarrow \\
& \mathfrak{S}_{1} \stackrel{\delta_{12}}{\longleftarrow} \mathfrak{S}_{3} \stackrel{\delta_{02}, \delta_{13}}{\longrightarrow} \quad \mathfrak{S}_{1} \times \mathfrak{S}_{1} \stackrel{\stackrel{s_{0} \times s_{0}}{\longleftarrow}}{\longleftarrow} \mathfrak{S}_{0} \times \mathfrak{S}_{0}
\end{aligned}
$$

Напомним, что в лемме 1.4.5(a) мы ввели объединения компонент связности $\mathfrak{S}_{1}^{\prime} \subset \mathfrak{S}_{1}$ и $\mathfrak{S}_{3}^{\prime} \subset \mathfrak{S}_{3}$ и показали, что $\delta_{12}\left(\mathfrak{S}_{3}^{\prime}\right)=\mathfrak{S}_{h e q} \cdot$

Аналогично определим $\left(\mathfrak{S}^{(1)}\right)_{0}^{\prime} \subset\left(\mathfrak{S}^{(1)}\right)_{0}$ как объединение компонент связности, пересекающихся с $s_{0}\left(\mathfrak{S}_{0}\right)$, и положим $\left(\mathfrak{S}^{(3)}\right)_{0}^{\prime}:=\delta_{02}^{-1}\left(\left(\mathfrak{S}^{(1)}\right)_{0}^{\prime}\right) \cap \delta_{13}^{-1}\left(\left(\mathfrak{S}^{(1)}\right)_{0}^{\prime}\right)$ $\subset\left(\mathfrak{S}^{(3)}\right)_{0}$.

Мы утверждаем, что $\delta_{12}\left(\left(\mathfrak{S}^{(3)}\right)_{0}^{\prime}\right)=\left(\mathfrak{S}^{(w e)}\right)_{0}$. Действительно, поскольку $s_{0}\left(\mathfrak{S}_{0}\right) \subset \mathfrak{S}^{(w e)}$, из предложения $2.2 .8(\mathrm{~d})$ следует, что $\delta_{02}(\widetilde{\phi}), \delta_{13}(\widetilde{\phi}) \in \mathfrak{S}^{(w e)}$ для любого $\widetilde{\phi} \in\left(\mathfrak{S}^{(3)}\right)_{0}^{\prime}$. Иными словами, если $\widetilde{\phi}$ соответствует диаграмме $E^{(0)} \stackrel{\phi_{1}}{\longrightarrow} E^{(1)} \stackrel{\phi_{2}}{\longrightarrow} E^{(2)} \stackrel{\phi_{3}}{\longrightarrow} E^{(3)}$, то $\phi_{2} \circ \phi_{1}$ и $\phi_{3} \circ \phi_{2}-$ слабые эквивалентности. Поэтому $\phi_{2}$ имеет левое и правое гомотопически обратные. Следовательно, $\phi_{2}$ - слабая эквивалентность, так что $\phi_{2}=\delta_{12}(\widetilde{\phi}) \in \mathfrak{S}^{(w e)}$.

Обратно, каждому $\phi \in \mathfrak{S}^{(w e)}$ соответствует гомотопическая эквивалентность $\phi: E^{(0)} \rightarrow E^{(1)}($ по лемме $1.3 .11(\mathrm{~b}))$, так что существует диаграмма $\widetilde{\phi}: E^{(1)} \stackrel{\phi^{\prime}}{\longrightarrow}$ 
${\underset{\sim}{\phi}}^{(0)} \stackrel{\phi}{\longrightarrow} E^{(1)} \stackrel{\phi^{\prime}}{\longrightarrow} E^{(0)}$, такая, что $\phi^{\prime} \circ \phi \sim \operatorname{Id}_{E^{(0)}}$ и $\phi \circ \phi^{\prime} \sim \operatorname{Id}_{E^{(1)}}$. По определению $\widetilde{\phi}$ соответствует элементу из $\left(\mathfrak{S}^{(3)}\right)_{0}^{\prime}$ и $\delta_{12}(\widetilde{\phi})=\phi$.

Теперь мы можем доказать утверждение теоремы. Поскольку $\psi^{(3)}$-слабая эквивалентность, индуцированное отображение $\pi_{0}\left(\left(\mathfrak{S}^{(3)}\right)_{0}\right) \rightarrow \pi_{0}\left(\mathfrak{S}_{3}\right)$ является биекцией. Далее, пользуясь определениями $\left(\mathfrak{S}^{(3)}\right)_{0}^{\prime}$ и $\mathfrak{S}_{3}^{\prime}$ и гомотопической коммутативностью среднего и правого внутренних квадратов диаграммы $(2.4)$, видим, что $\psi^{(3)}$ индуцирует биекцию $\pi_{0}\left(\left(\mathfrak{S}^{(3)}\right)_{0}^{\prime}\right) \rightarrow \pi_{0}\left(\mathfrak{S}_{3}^{\prime}\right)$. Наконец, поскольку $\mathfrak{S}_{h e q}=\delta_{12}\left(\mathfrak{S}_{3}^{\prime}\right),\left(\mathfrak{S}^{(w e)}\right)_{0}=\delta_{12}\left(\left(\mathfrak{S}^{(3)}\right)_{0}^{\prime}\right)$ и левый внутренний квадрат диаграммы (2.4) гомотопически коммутативен, $\psi^{(1)}$ индуцирует биекцию $\pi_{0}\left(\left(\mathfrak{S}^{(w e)}\right)_{0}\right) \rightarrow \pi_{0}\left(\mathfrak{S}_{\text {heq }}\right)$.

\section{3. Вложение Йонеды.}

2.3.1. Противоположное симплициальное пространство. (а) Для любого отображения $\tau:[n] \rightarrow[m]$ в $\Delta$ обозначим через $\iota(\tau):[n] \rightarrow[m]$ отображение $\iota(\tau)(n-i):=m-\tau(i)$. Тогда $\iota$ определяет функтор $\Delta \rightarrow \Delta$, а значит, и функтор $\iota^{*}: s S p=\operatorname{Hom}\left(\Delta^{o p}, S p\right) \rightarrow \operatorname{Hom}\left(\Delta^{o p}, S p\right)=s S p$.

(b) Для любого $X \in s S p$ положим $X^{o p}:=\iota^{*}(X) \in s S p$. Явным образом, $\left(X^{o p}\right)_{n}=X_{n}$ для всех $n$, и для любого $\tau:[n] \rightarrow[m]$ отображение $\tau^{*}:\left(X^{o p}\right)_{m} \rightarrow$ $\left(X^{o p}\right)_{n}$ - это отображение $\iota(\tau)^{*}: X_{m} \rightarrow X_{n}$.

(c) Заметим, что если $X-$ (полное) пространство Сигала, то таково же и $X^{o p}$ и имеется равенство гомотопических категории $\mathrm{Ho}\left(X^{o p}\right)=(\mathrm{Ho} X)^{o p}$. Поэтому назовем $X^{\text {ор }}$ противоположным симплиииальным пространством.

2.3.2. Скрученная категория стрелок. (а) Рассмотрим функтор $\mu: \Delta \rightarrow$ $\Delta$, такой, что $\mu([n])=[2 n+1]$ и для любого $\tau:[n] \rightarrow[m]$ из $\Delta$ отображение $\mu(\tau):[2 n+1] \rightarrow[2 m+1]$ определено формулами $\mu(\tau)(n-i):=m-\tau(i)$ и $\mu(\tau)(n+1+j)=(m+1+\tau(j))$ при $i, j=0, \ldots, n$.

(b) Для любого $X \in s S p$ определим симплициальное пространство $\mathscr{M}(X):=$ $\mu^{*}(X) \in s S p$. Явным образом, $\mathscr{M}(X)_{n}=X_{2 n+1}$ для всех $n$ и для любого $\tau:[n] \rightarrow[m]$ отображение $\tau^{*}: \mathscr{M}(X)_{m} \rightarrow \mathscr{M}(X)_{n}$ - это отображение $\mu(\tau)^{*}:$ $X_{2 m+1} \rightarrow X_{2 n+1}$.

(c) Имеются естественные морфизмы $\iota \rightarrow \mu$ и $\mathrm{Id} \rightarrow \mu$ функторов $\operatorname{Hom}(\Delta, \Delta)$, которые соответствуют отображениям $e^{0}:[n] \rightarrow[2 n+1]$ и $e^{n+1}:[n] \rightarrow[2 n+1]$ соответственно. Эти отображения соответствуют морфизму $\pi_{X}: \mathscr{M}(X) \rightarrow X^{o p} \times X$ B $s S p$.

2.3.3. Замечания. (а) Заметим, что категория $\Delta$ эквивалентна категории $\Delta^{\prime}$ конечных линейно упорядоченных множеств, а функторы $\iota, \mu: \Delta \rightarrow \Delta$ соответствуют функторам $\iota, \mu: \Delta^{\prime} \rightarrow \Delta^{\prime}$, определенным формулами $\iota(P)=P^{o p}$ и $\mu(P)=P^{o p} * P, \ll$ соединение» $P^{o p}$ и $P$.

(b) Можно показать (пользуясь леммой 2.3.4 и 2.1.4(b)), что если $X-$ (полное) пространство Сигала, то $\mathscr{M}(X)$ - также (полное) пространство Сигала. В этом случае пространство объектов $\mathscr{M}(X)_{0}$ совпадает с пространством морфизмов $X_{1}$ и для любых $\alpha: x \rightarrow y$ и $\alpha^{\prime}: x^{\prime} \rightarrow y^{\prime}$ из $\mathscr{M}(X)_{0}=X_{1}$ пространство отображений $\operatorname{map}_{\mathscr{M}(X)}\left(\alpha, \alpha^{\prime}\right)$ можно интуитивно понимать как пространство троек $\left(\beta, \beta^{\prime}, \gamma\right)$, где $\beta: x^{\prime} \rightarrow x$ и $\beta^{\prime}: y \rightarrow y^{\prime}$ принадлежат $X_{1}$, а $\gamma$-путь, соединяющий $\beta^{\prime} \circ \alpha \circ \beta$ и $\alpha^{\prime}$.

Начиная с этого места, в данном параграфе всегда предполагается, что $X-$ пространство Сигала. 
2.3.4. Лемма. Отображсение $\pi_{X}: \mathscr{M}(X) \rightarrow X^{o p} \times X-$ левое расслоение.

Доказательство. Чтобы показать, что $\pi_{X}$ - расслоение, нужно проверить, что индуцированное отображение

$$
\mathscr{M}(X)_{n} \rightarrow \mathscr{M}(X)_{\partial n} \times\left(X^{o p} \times X\right)_{\partial n}\left(X^{o p} \times X\right)_{n}
$$

является расслоением для любого $n \geqslant 0$. Напомним, что $\mathscr{M}(X)_{n}=X_{2 n+1}=$ $\operatorname{Map}(F[2 n+1], X)$. Поскольку $\partial F[n]=\bigcup_{i=0}^{n} d^{i} F[n-1]$, получаем, что $\mathscr{M}(X)_{\partial n}=$ $\operatorname{Map}\left(F[2 n+1]^{\prime}, X\right)$, где $F[2 n+1]^{\prime} \subset F[2 n+1]$ - объединение $\bigcup_{i=0}^{n} d^{i, 2 n+1-i}(F[2 n-$ 1]) $\subset F[2 n+1]$. Таким образом, морфизм $(2.5)$ можно отождествить с морфизMOM

$\operatorname{Map}(F[2 n+1], X) \rightarrow \operatorname{Map}\left(F[2 n+1]^{\prime} \sqcup_{\left(e^{0} \partial F[n] \cup e^{n+1} \partial F[n]\right)}\left(e^{0} F[n] \cup e^{n+1} F[n]\right), X\right)$. Поскольку $F[2 n+1]^{\prime} \cap\left(e^{0} F[n] \cup e^{n+1} F[n]\right)=e^{0} \partial F[n] \cup e^{n+1} \partial F[n] \subset F[2 n+1]$, естественное отображение $F[2 n+1]^{\prime} \sqcup_{\left(e^{0} \partial F[n] \cup e^{n+1} \partial F[n]\right)}\left(e^{0} F[n] \cup e^{n+1} F[n]\right) \rightarrow$ $F[2 n+1]$ является корасслоением. Так как $X$ фибрантно, то отображение $(2.5)-$ расслоение.

Остается показать, что расслоение $\mathscr{M}(X)_{n} \rightarrow \mathscr{M}(X)_{0} \times\left(X^{o p} \times X\right)_{0}\left(X^{o p} \times X\right)_{n}$, или, что эквивалентно, $X_{2 n+1} \rightarrow X_{n} \times_{X_{0}} X_{1} \times_{X_{0}} X_{n},-$ слабая эквивалентность. Поскольку $X$ - пространство Сигала, так что и композиция

$X_{2 n+1} \rightarrow X_{n} \times_{X_{0}} X_{1} \times_{X_{0}} X_{n} \rightarrow\left(X_{1} \times_{X_{0}} \cdots \times_{X_{0}} X_{1}\right) \times_{X_{0}} X_{1} \times_{X_{0}}\left(X_{1} \times_{X_{0}} \cdots \times_{X_{0}} X_{1}\right)$,

и второй морфизм являются тривиальными расслоениями, это следует из свойства «2 из 3 ».

2.3.5. Замечание. Из леммы 2.3.4 и леммы 2.1.2(a) следует, что левое расслоение $\pi_{X}$ индуцирует левое расслоение $\{x\} \times_{X}$ ор $\mathscr{M}(X) \rightarrow X$ для любого $x \in X$. Заметим, что $\left(\{x\} \times_{X} \text { op } \mathscr{M}(X)\right)_{0}=\{x\} \times_{X_{0}} X_{1}=(x \backslash X)_{0}$.

2.3.6. Лемма. Пусть $X$ - пространство Сигала $u x \in X$. Существует слабая эквивалентность $\widetilde{\phi}: x \backslash X \rightarrow\{x\} \times_{X}$ ор $\mathscr{M}(X)$ левых расслоений над $X$, mакая, чmо $\widetilde{\phi}\left(\mathrm{id}_{x}\right) \sim \operatorname{id}_{x}$.

Доказательство. Достаточно построить гомотопическую эквивалентность $\psi:\{x\} \times_{X}$ ор $\mathscr{M}(X) \rightarrow x \backslash X$ над $X$, такую, что $\psi\left(\operatorname{id}_{x}\right) \sim \mathrm{id}_{x}$, и взять в качестве $\widetilde{\phi}$ ее гомотопически обратную.

Чтобы построить $\psi$, мы построим симплициальное пространство $\widetilde{x \backslash X}$ над $X$ и отображения $\psi^{\prime}: \widetilde{x \backslash X} \rightarrow x \backslash X$ и $\psi^{\prime \prime}: \widetilde{x \backslash X} \rightarrow\{x\} \times_{X^{\circ}} \mathscr{\mathscr { M }}(X)$ над $X$, такие, что $(\widetilde{x \backslash X})_{0}=(x \backslash X)_{0}, \psi_{0}^{\prime}=\psi_{0}^{\prime}=\mathrm{Id}$, а $\psi^{\prime}$ - тривиальное корасслоение. Поскольку $x \backslash X \rightarrow X-$ расслоение, отображение $\psi^{\prime \prime}$ расширяется до отображения $\psi:\{x\} \times_{X \text { ор }} \mathscr{M}(X) \rightarrow x \backslash X$ над $X$.

В этом случае $\psi_{0}=\mathrm{Id}$ должно быть слабой эквивалентностью, так что $\psi$ будет слабой эквивалентностью по лемме 2.1.5, а значит, и гомотопической эквивалентностью по лемме 1.3.11(b).

Для любого отображения $\tau:[n] \rightarrow[m]$ обозначим через $\tau^{\prime}$ отображение $[n+$ $1] \rightarrow[m+1]$ определенное формулами $\tau^{\prime}(0)=0$ и $\tau^{\prime}(i+1)=\tau(i)+1$ для всех $i=0, \ldots, n$. Рассмотрим $\widehat{x \backslash X} \in s S p$, такое, что $(\widehat{x \backslash X})_{n}:=\{x\} \times_{X_{0}, \delta_{0}} X_{n+1}$ и для любого $\tau$ отображение $\tau^{*}:(\widehat{x \backslash X})_{m} \rightarrow(\widehat{x \backslash X})_{n}$ индуцировано отображением $\tau^{\prime *}: X_{m+1} \rightarrow X_{n+1}$. 
Заметим, что проекции $e_{1}: X_{n+1} \rightarrow X_{n}$ индуцируют проекцию $\widetilde{x \backslash X} \rightarrow X$. Далее, отображения $[n] \times[1] \rightarrow[n+1]:(i, j) \mapsto(i+j) j$ индуцируют отображения $F[n] \times F[1] \rightarrow F[n+1]$ и

$$
p_{n}: X_{n+1}=\operatorname{Map}(F[n+1], X) \rightarrow \operatorname{Map}(F[n] \times F[1], X)=\left(X^{F[1]}\right)_{n} .
$$

Кроме того, отображения $p_{n}$ индуцируют отображение $\psi^{\prime}: \widetilde{x \backslash X} \rightarrow x \backslash X$ над $X$.

Наконец, отображения $r:[2 n+1] \rightarrow[n+1]$, определенные формулами $r(i)=0$ и $r(i+n+1)=i+1$ для всех $i=0, \ldots, n$, индуцируют отображения $X_{n+1} \rightarrow$ $X_{2 n+1}$ и задают отображение $\psi^{\prime \prime}: \widehat{x \backslash X} \rightarrow\{x\} \times_{X}$ ор $\mathscr{M}(X)$ над $X$, которое, как мы утверждаем, является тривиальным корасслоением.

Нам нужно доказать, что $\psi_{n}^{\prime \prime}:\{x\} \times_{X_{0}} X_{n+1} \rightarrow\{x\} \times_{X_{n}} X_{2 n+1}$ - тривиальное корасслоение для всех $n$. Поскольку $X$ - пространство Сигала, естественное отображение $X_{2 n+1} \rightarrow X_{n} \times_{X_{0}} X_{n+1}$ есть тривиальное расслоение, пулбэк $\pi_{n}:\{x\} \times_{X_{n}} X_{2 n+1} \rightarrow\{x\} \times_{X_{0}} X_{n+1}$ которого - тривиальное расслоение, удовлетворяющее соотношению $\pi_{n} \circ \psi_{n}^{\prime \prime}=\mathrm{Id}$. Поэтому $\psi_{n}^{\prime \prime}:\{x\} \times_{X_{0}} X_{n+1} \rightarrow\{x\} \times_{X_{n}}$ $X_{2 n+1}$ - тривиальное корасслоение в силу свойства «2 из $3 »$, что и завершает доказательство.

2.3.7. Вложение Йонеды. Зафиксируем бесконечное множество $\mathscr{U}$ и положим $\mathfrak{S}:=\mathfrak{S}_{\mathscr{U}}$.

(а) Для любого $X \in s S p_{\mathscr{U}}$ положим $\mathfrak{P}(X):=\mathfrak{S}^{X^{o p}}$. Поскольку $\mathfrak{S}-$ полное пространство Сигала, $\mathfrak{P}(X)$ также есть полное пространство Сигала (в силу 1.4.6), и мы назовем его $\infty$-категорией симплициальных предпучков на $X$.

(b) По определению пространства $\mathfrak{S}$ для любого пространства Сигала $X$ левому расслоению $\pi_{X}: \mathscr{M}(X) \rightarrow X^{o p} \times X$ соответствует морфизм $X^{o p} \times X \rightarrow \mathfrak{S}$, а значит, и морфизм $j_{X}: X \rightarrow \mathfrak{P}(X)$. Назовем $j_{X}$ вложением Йонедъ пространства $X$.

(c) Для любых $x \in X$ и $\alpha \in \mathfrak{P}(X)$ рассмотрим $\alpha(x) \in \mathfrak{S}$ и обозначим через $E_{\alpha(x)} \rightarrow \mathrm{pt}$ соответствующее левое расслоение.

2.3.8. Пример. По определению для любого $x \in X$ элемент $j_{X}(x) \in \mathfrak{P}(X)=$ $\mathfrak{S}^{X^{o p}}$ соответствует левому расслоению $\mathscr{M}(X) \times_{X}\{x\}=\{x\} \times_{X} \mathscr{M}\left(X^{o p}\right) \rightarrow X^{o p}$.

2.3.9. Теорема. Пусть $X$ - пространство Сигала, $x \in X u \alpha \in \mathfrak{P}(X)$.

(а) Имеется «естественная» слабая эквивалентность $\operatorname{map}_{\mathfrak{P}(X)}\left(j_{X}(x), \alpha\right) \rightarrow$ $\left(E_{\alpha(x)}\right)_{0}$.

(b) Вложение Йонедъ $j_{X}: X \rightarrow \mathfrak{P}(X)$ является вполне строгим (см. 1.4.2(d)).

Сначала введем некоторые обозначения.

2.3.10. Универсальное левое расслоение над $\boldsymbol{X}^{o p}$. (а) Пусть $\widetilde{E} \rightarrow$ $X^{o p} \times \mathfrak{P}(X)$ - левое расслоение, соответствующее естественному отображению $\mathrm{ev}_{X^{o p}}: X^{o p} \times \mathfrak{P}(X) \rightarrow \mathfrak{S}$. Тогда для любого $\alpha \in \mathfrak{P}(X)$ пулбэк $\widetilde{E}_{\alpha}:=\widetilde{E} \times_{\mathfrak{P}(X)}\{\alpha\}$ есть левое расслоение над $X^{o p}$, соответствующее $\alpha$. В частности, для любого $x \in X$ его слой $\left(\widetilde{E}_{\alpha}\right)_{x}$ есть левое расслоение $E_{\alpha(x)} \rightarrow \mathrm{pt}$, соответствующее $\alpha(x) \in \mathfrak{S}$ (см. 2.3.7(c)).

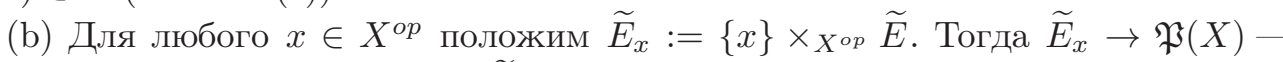
левое расслоение, такое, что $\left(\widetilde{E}_{x}\right)_{\alpha}=E_{\alpha(x)}$ для любого $\alpha \in \mathfrak{P}(X)$ (в силу $\left(\right.$ a)). Для любого $y \in X$ имеет место равенство $\left(\widetilde{E}_{x}\right)_{j_{X}(y)}=E_{j_{X}(y)(x)}$, так что 
$\left(\left(\widetilde{E}_{x}\right)_{j_{X}(y)}\right)_{0}=\operatorname{map}_{X}(x, y)$. В частности, имеется элемент $\operatorname{id}_{x} \in \operatorname{map}_{X}(x, x)$ из $\left(\widetilde{E}_{x}\right)_{j_{X}(x)}$.

(c) По определению отображение $X^{o p} \times X \rightarrow \mathfrak{S}$, соответствующее левому расслоению $\pi_{X}: \mathscr{M}(X) \rightarrow X^{o p} \times X$, представляется в виде композиции отображений $\operatorname{Id} \times j_{X}: X^{o p} \times X \rightarrow X^{o p} \times \mathfrak{P}(X)$ и еv $X^{o p}$. Поэтому $\mathscr{M}(X)=\widetilde{E} \times \mathfrak{P}(X) X$, так что $\widetilde{E}_{x} \times_{\mathfrak{P}(X)} X=\{x\} \times_{X^{\text {op }}} \mathscr{M}(X)$.

2.3.11. Замечания. Гомотопическая эквивалентность $\psi^{(1)}: \mathfrak{S}^{F[1]} \rightarrow \mathfrak{S}^{(1)}$ над $\mathfrak{S}^{2}$ из предложения 2.2.9(b) индуцирует гомотопическую эквивалентность из $\mathfrak{P}(X)^{F[1]}=\left(\mathfrak{S}^{F[1]}\right)^{X^{o p}}$ в $\mathfrak{P}(X)^{(1)}:=\left(\mathfrak{S}^{(1)}\right)^{X^{\text {оp }}}$ над $\mathfrak{P}(X)^{2}$. Поэтому для любого $\alpha \in \mathfrak{P}(X)$ она индуцирует гомотопическую эквивалентность $\psi_{\alpha}: \alpha \backslash \mathfrak{P}(X) \rightarrow$ $(\alpha \backslash \mathfrak{P}(X))^{\prime}:=\{\alpha\} \times_{\mathfrak{P}(X)} \mathfrak{P}(X)^{(1)}$ над $\mathfrak{P}(X)$. Тогда в силу предложения 2.2.9(c) имеем $\psi_{\alpha}\left(\operatorname{id}_{\alpha}\right) \sim \operatorname{id}_{\alpha}$.

2.3.12. Лемма. Для любого $x \in X$ существует слабая эквивалентность $\phi: j_{X}(x) \backslash \mathfrak{P}(X) \rightarrow \widetilde{E}_{x}$ левых расслоений над $\mathfrak{P}(X)$, такая, что $\phi\left(\operatorname{id}_{j_{X}(x)}\right) \sim$ $\operatorname{id}_{x} \in\left(\widetilde{E}_{x}\right)_{j_{X}(x)}$.

Доказательство. Построим $\phi$ как композицию трех слабых эквивалентностей над $\mathfrak{P}(X)$ :

$$
j_{X}(x) \backslash \mathfrak{P}(X) \stackrel{\phi^{\prime \prime \prime}}{\longrightarrow}\left(j_{X}(x) \backslash \mathfrak{P}(X)\right)^{\prime} \stackrel{\phi^{\prime \prime}}{\longrightarrow}\left(\left(x \backslash X^{o p}\right) \backslash \mathfrak{P}(X)\right)^{\prime} \stackrel{\phi^{\prime}}{\longrightarrow} \widetilde{E}_{x} .
$$

По определению, если $\alpha: K \rightarrow \mathfrak{P}(X)$ соответствует левому расслоению $G \rightarrow$ $X^{o p} \times K$, то отображения $K \rightarrow\left(\left(x \backslash X^{o p}\right) \backslash \mathfrak{P}(X)\right)^{\prime}$ над $\alpha$ находятся во взаимно однозначном соответствии с отображениями $\nu:\left(x \backslash X^{o p}\right) \times K \rightarrow G$ над $X^{o p} \times K$, в то время как отображения $K \rightarrow\left(j_{X}(x) \backslash \mathfrak{P}(X)\right)^{\prime}$ над $\alpha$ находятся во взаимно однозначном соответствии с отображениями $\nu^{\prime}:\left(\{x\} \times_{X} \mathscr{M}\left(X^{o p}\right)\right) \times K \rightarrow G$ над $X^{o p} \times K$ (мы воспользовались 2.3.8).

Пусть $\widetilde{\phi}: x \backslash X^{o p} \rightarrow\{x\} \times_{X} \mathscr{M}\left(X^{o p}\right)$ - гомотопическая эквивалентность из леммы 2.3.6; определим $\phi^{\prime \prime}$ как отображение, которое переводит $\nu^{\prime}$ в $\nu^{\prime} \circ \widetilde{\phi}$. Тогда $\phi^{\prime \prime}$ - гомотопическая эквивалентность, потому что таково $\widetilde{\phi}$.

Далее заметим, что отображения $K \rightarrow \widetilde{E}_{x}$ над $\alpha: K \rightarrow \mathfrak{P}(X)$ находятся во взаимно однозначном соответствии с сечениями $s$ левого расслоения $G_{x}:=$ $\{x\} \times_{X^{\text {op }}} G \rightarrow K$. Определим $\phi^{\prime}$ как отображение, переводящее $\nu:\left(x \backslash X^{o p}\right) \times K \rightarrow$ $G$ в $s:=\left.\nu\right|_{\operatorname{id}_{x}}: K \rightarrow G_{x}$.

Поскольку $\phi^{\prime}$ - отображение левых расслоений, чтобы доказать, что $\phi^{\prime}-$ слабая эквивалентность, остается показать, что для любого $\alpha \in \mathfrak{P}(X)$ индуцированное отображение $\left(\phi_{\alpha}^{\prime}\right)_{0}$ есть слабая эквивалентность симплициальных множеств (по лемме 2.1.5). Пусть $G \rightarrow X^{o p}$ - левое расслоение, соответствующее $\alpha$. Тогда $\left(\phi_{\alpha}^{\prime}\right)_{0}$ - это тривиальное расслоение $\operatorname{evid}_{x}: \operatorname{Map}_{X^{o p}}\left(x \backslash X^{o p}, G\right) \rightarrow$ $\left(G_{x}\right)_{0}$ из предложения 2.1.7.

Наконец, определим $\phi^{\prime \prime \prime}: j_{X}(x) \backslash \mathfrak{P}(X) \rightarrow\left(j_{X}(x) \backslash \mathfrak{P}(X)\right)^{\prime}$ как слабую эквивалентность из 2.3.11 и положим $\phi:=\phi^{\prime} \circ \phi^{\prime \prime} \circ \phi^{\prime \prime \prime}$. По построению $\phi^{\prime \prime \prime}\left(\operatorname{id}_{j_{X}(x)}\right) \sim$ $\operatorname{id}_{j_{X}(x)}, \phi^{\prime \prime}\left(\operatorname{id}_{j_{X}(x)}\right)=\widetilde{\phi}$ и $\phi^{\prime}(\widetilde{\phi})=\widetilde{\phi}\left(\operatorname{id}_{x}\right) \sim \operatorname{id}_{x}$. Следовательно, $\phi\left(\operatorname{id}_{j_{X}(x)}\right) \sim$ $\operatorname{id}_{x}$.

Теперь мы готовы доказать лемму Йонеды. 
Доказательство теоремы 2.3.9. (а) Согласно лемме 2.3.12, существует гомотопическая эквивалентность $\phi: j_{X}(x) \backslash \mathfrak{P}(X) \rightarrow \widetilde{E}_{x}$ левых расслоений над $\mathfrak{P}(X)$, такая, что $\phi\left(\operatorname{id}_{j_{X}(x)}\right) \sim \mathrm{id}_{x}$. Поскольку для любого $\alpha \in \mathfrak{P}(X)$ слой расслоения $\widetilde{E}_{x}$ в точке $\alpha$ есть $E_{\alpha(x)}$ (см. 2.3.10(b)), $\phi$ индуцирует гомотопическую эквивалентность $\phi_{\alpha}: \operatorname{map}_{\mathfrak{P}(X)}\left(j_{X}(x), \alpha\right) \rightarrow\left(E_{\alpha(x)}\right)_{0}$.

(b) Морфизм $j_{X}: X \rightarrow \mathfrak{P}(X)$ индуцирует морфизм $x \backslash X \rightarrow j_{X}(x) \backslash \mathfrak{P}(X)$. Поэтому $j_{X}$ индуцирует морфизм $\psi: x \backslash X \rightarrow j_{X}(x) \backslash \mathfrak{P}(X) \times_{\mathfrak{P}(X)} X$ левых расслоений над $X$. Утверждается, что $\psi$-слабая эквивалентность, так что она индуцирует слабую эквивалентность слоев $\psi_{b}: \operatorname{map}_{X}(a, b) \rightarrow \operatorname{map}_{\mathfrak{P}(X)}\left(j_{X}(a), j_{X}(b)\right)$ для всех $b \in X$.

Рассмотрим композицию $\phi \circ \psi: x \backslash X \rightarrow \widetilde{E}_{x} \times_{\mathfrak{P}(X)} X$, где $\phi$ такой же, как и в доказательстве п. (а). Поскольку $\widetilde{E}_{x} \times_{\mathfrak{P}(X)} X=\{x\} \times_{X}$ ор $\mathscr{M}(X)$ (см. 2.3.10(c)), $\phi \circ \psi$ - это отображение $x \backslash X \rightarrow\{x\} \times_{X^{\circ p}} \mathscr{M}(X)$ над $X$, которое по построению удовлетворяет соотношению $\phi \circ \psi\left(\mathrm{id}_{x}\right) \sim \mathrm{id}_{x}$. Поэтому $\phi \circ \psi-$ слабая эквивалентность в силу следствия 2.1.8 и леммы 2.3.6, так что $\psi$-слабая эквивалентность в силу свойства $\ll 2$ из $3 »$.

\section{§3. Квазирасслоения симплициальных пространств}

\section{1. Определения и основные свойства.}

3.1.1. Определение. Пусть $\mathscr{C}$ - правильная справа модельная категория. Будем говорить, что морфизм $p: X \rightarrow B$ в $\mathscr{C}$ является квазирасслоением, если для любой слабой эквивалентности $g: Y \rightarrow Z$ над $B$ ее пулбэк $p^{*}(g): p^{*}(Y) \rightarrow$ $p^{*}(Z)$ (см. 1.1.1(d)) - слабая эквивалентность над $X$.

3.1.2. Замечания. (а) По определению произвольный пулбэк квазирасслоения есть квазирасслоение и композиция квазирасслоений - также квазирасслоение.

(b) В случае когда $\mathscr{C}$ - модельная категория топологических пространств, наше понятие квазирасслоения сильнее, чем классическое. Однако мы будем использовать это понятие только для модельных категорий пространств и симплициальных пространств, где классического понятия не существует.

(c) После того, как эта статья была по существу уже написана, мы обнаружили, что квазирасслоения изучал также Резк в неопубликованном препринте [8], где они называются острыми (sharp) морфизмами. Наша терминология кажется нам более содержательной.

3.1.3. Лемма. (а) Каждое расслоение из $\mathscr{C}$ является квазирасслоением.

(b) Пусть $f: X \rightarrow X^{\prime}$ - слабая эквивалентность квазирасслоений $p: X \rightarrow B$ и $p^{\prime}: X^{\prime} \rightarrow B$ над $B$. Тогда для любого морфизма $\tau: A \rightarrow B$ пулбэк $\tau^{*}(f)$ : $X \times_{B} A \rightarrow X^{\prime} \times{ }_{B} A$ является слабой эквивалентностью.

(c) Обратно, предположим, что $f: X \rightarrow X^{\prime}$ - слабая эквивалентность над $B$, такая, что каждый пулбэк $\tau^{*}(f)$ является слабой эквивалентностью. Тогда $p: X \rightarrow B$ является квазирасслоением в том и только в том случае, когда $p^{\prime}: X^{\prime} \rightarrow B-$ квазирасслоение.

Доказательство. Утверждение (а) следует из того факта, что $\mathscr{C}$ правильна справа. 
(b) Если $\tau: A \rightarrow B-$ расслоение, то $\tau^{*}(g)$ - слабая эквивалентность в силу п. (а). Если $\tau$ - слабая эквивалентность, то $\tau^{*}(X) \rightarrow X$ и $\tau^{*}\left(X^{\prime}\right) \rightarrow X^{\prime}-$ слабые эквивалентности, потому что $X \rightarrow B$ и $X^{\prime} \rightarrow B$ являются квазирасслоениями. Поэтому $\tau^{*}(f): \tau^{*}(X) \rightarrow \tau^{*}\left(X^{\prime}\right)$ - слабая эквивалентность в силу свойства «2 из 3». Поскольку каждый морфизм разлагается в композицию тривиального корасслоения и расслоения, отсюда следует и общий случай.

(с) Пусть $g: Y \rightarrow Z$ - слабая эквивалентность над $B$. Тогда $X \times_{B} Y \rightarrow$ $X^{\prime} \times_{B} Y$ и $X \times_{B} Z \rightarrow X^{\prime} \times_{B} Z-$ слабые эквивалентности в силу предположения относительно $f$. Поэтому в силу свойства «2 из $3 » p^{*}(g): X^{\prime} \times{ }_{B} Y \rightarrow X^{\prime} \times{ }_{B} Z$ является слабой эквивалентностью тогда и только тогда, когда $p^{*}(g)-$ слабая эквивалентность. Таким образом, по определению $p: X \rightarrow B$ является квазирасслоением тогда и только тогда, когда $p^{\prime}: X^{\prime} \rightarrow B-$ квазирасслоение.

3.1.4. Следствие. Предположим, что модельная категория $\mathscr{C}$ обладает тем свойством, что пулбэк корасслоения является корасслоением, и пусть $p^{\prime}: X^{\prime} \rightarrow B-\oint$ фирантная замена отображения $p: X \rightarrow B$. Тогда $p$ является квазирасслоением в том и только в том случае, когда $\tau^{*}\left(p^{\prime}\right)$ - фибрантная замена отображения $\tau^{*}(p)$ для любого $\tau: A \rightarrow B$.

Доказательство. Согласно аксиоме CM5, p разлагается в композицию $X \stackrel{i}{\longrightarrow} X^{\prime} \stackrel{p^{\prime}}{\longrightarrow} B$, где $i$ - тривиальное корасслоение, а $p$ - расслоение. Тогда для любого отображения $\tau: A \rightarrow B$ отображение $\tau^{*}\left(p^{\prime}\right)$ есть расслоение, в то время как $\tau^{*}(i)$ - корасслоение. Таким образом, нужно доказать, что $X \rightarrow B$ является квазирасслоением тогда и только тогда, когда каждое $\tau^{*}(i)-$ слабая эквивалентность. Поскольку $p^{\prime}: X^{\prime} \rightarrow B$ - квазирасслоение по лемме 3.1.3(a), нужное утверждение следует из леммы 3.1.3(b),(c).

3.1.5. Следствие. Пусть дана коммутативная диаграмма

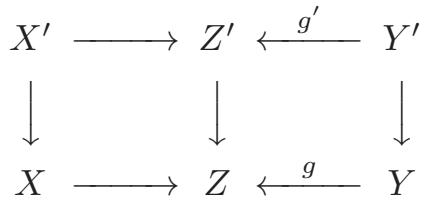

где g и $g^{\prime}$ - квазирасслоения и все вертикальные морфизмы - слабые эквивалентности. Тогда индуцированное отображение $X^{\prime} \times Z_{Z^{\prime}} Y^{\prime} \rightarrow X \times \times_{Y} Z-$ слабая эквивалентность.

Доказательство. Слабая эквивалентность $Y^{\prime} \rightarrow Y$ разлагается в композицию отображений $Y^{\prime} \rightarrow Z^{\prime} \times_{Z} Y \rightarrow Y$, второе из которых - слабая эквивалентность, потому что оно является пулбэком слабой эквивалентности $Z^{\prime} \rightarrow Z$ вдоль квазирасслоения $Y \rightarrow Z$. Поэтому в силу свойства «2 из $3 » Y^{\prime} \rightarrow Z^{\prime} \times_{Z} Y-$ слабая эквивалентность квазирасслоений над $Z^{\prime}$.

Теперь отображение $X^{\prime} \times_{Z^{\prime}} Y^{\prime} \rightarrow X \times_{Y} Z$ разлагается в композицию отображений

$$
X^{\prime} \times{ }_{Z^{\prime}} Y^{\prime} \rightarrow X^{\prime} \times{ }_{Z^{\prime}}\left(Z^{\prime} \times_{Z} Y\right)=X^{\prime} \times_{Z} Y \rightarrow X \times_{Z} Y,
$$

первое из которых является слабой эквивалентностью как пулбэк слабой эквивалентности квазирасслоений (мы воспользовались леммой 3.1.3(b)), в то время как второе есть слабая эквивалентность, потому что $Y \rightarrow Z$ - квазирасслоение, а $X^{\prime} \rightarrow X-$ слабая эквивалентность. 
Начиная с этого места, предположим, что $\mathscr{C}$ - либо категория $S p$ с модельной структурой Кана (теорема 1.2.6), либо категория $s S p$ с модельной структурой Риди (теорема 1.3.6).

3.1.6. Лемма. Пусть $X_{B} \rightarrow B$ - квазирасслоение в $s S p, A \rightarrow B$ - корасслоение, а $i: X_{A}:=X_{B} \times_{B} A \rightarrow Y_{A}$ - слабая эквивалентность квазирасслоений над $A$. Тогда пушаут $Y_{B}:=X_{B} \sqcup_{X_{A}} Y_{A}-$ квазирасслоение над $B \sqcup_{A} A=B$, a естественное отображение $j: X_{B} \rightarrow Y_{B}-$ слабая эквивалентность.

Доказательство. Поскольку $X_{B} \rightarrow B$-квазирасслоение, достаточно показать, что для любого отображения $\tau: B^{\prime} \rightarrow B$ пулбэк $\tau^{*}(j)$ является слабой эквивалентностью (по лемме 3.1.3(с)).

Заметим, что $\tau^{*}(j)$ - пушаут отображения $\tau^{*}(i): \tau^{*}\left(X_{A}\right) \rightarrow \tau^{*}\left(Y_{A}\right)$ вдоль $\tau^{*}\left(X_{A}\right) \rightarrow \tau^{*}\left(X_{B}\right)$. Поскольку $A \rightarrow B$ - корасслоение, индуцированные отображения $X_{A} \rightarrow X_{B}$ и $\tau^{*}\left(X_{A}\right) \rightarrow \tau^{*}\left(X_{B}\right)$ являются корасслоениями. Так как $i-$ слабая эквивалентность квазирасслоений, то $\tau^{*}(i)$ - слабая эквивалентность по лемме 3.1.3(b). Поэтому пушаут $\tau^{*}(j)$ - слабая эквивалентность, ибо категория $s S p$ правильна слева.

3.1.7. Лемма. Морфизм $p: X \rightarrow B$ является квазирасслоением в $s S p$ тогда и только тогда, когда $p_{n}: X_{n} \rightarrow B_{n}-$ квазирасслоение в $S p$ для любого $n$.

Доказательство. Заметим, что если $p_{n}$ - квазирасслоение для всех $n$, то для любой слабой эквивалентности $g: Y \rightarrow Z$ над $B$ соответствующие отображения $g_{n}: Y_{n} \rightarrow Z_{n}$ являются слабыми эквивалентностями над $B_{n}$ для всех $n$. Поэтому каждое $p^{*}(g)_{n}=p_{n}^{*}\left(g_{n}\right)$ - слабая эквивалентность, ибо $p_{n}$-квазирасслоение. Следовательно, $p^{*}(g)$ есть слабая эквивалентность, так что $p$-квазирасслоение.

Обратно, предположим, что $p$-квазирасслоение. Каждая слабая эквивалентность $\widetilde{g}: \widetilde{Y} \rightarrow \widetilde{Z}$ над $B_{n}$ определяет слабую эквивалентность $g:=\widetilde{g} \times$ $\operatorname{Id}_{F[n]}: \widetilde{Y} \times F[n] \rightarrow \widetilde{Z} \times F[n]$ над $B \times F[n]$, такую, что $\widetilde{g}-$ сужение отображения $g_{n}$ на $\operatorname{Id}_{F[n]} \in F[n]_{n}$. Так как $p$ - квазирасслоение, то пулбэк $p^{*}(g)$ - слабая эквивалентность над $X \times F[n]$. Таким образом, $p_{n}^{*}(\widetilde{g})=\left(p^{*}(g)_{n}\right)_{\operatorname{Id}_{F[n]}}-$ слабая эквивалентность, откуда следует, что $p_{n}-$ квазирасслоение.

3.1.8. Определение. Отображение $f: X \rightarrow B$ в $s S p$ называется левым квазирасслоением, если $f$-квазирасслоение и морфизм $X_{n} \rightarrow X_{0} \times_{B_{0}} B_{n}$, индуцированный включением $\delta^{0}:[0] \hookrightarrow[n],-$ слабая эквивалентность для всех $n$.

3.1.9. Замечание. Из леммы 2.1.3(с) следует, что расслоение $f: X \rightarrow B$ в $s S p$ есть левое расслоение тогда и только тогда, когда оно является левым квазирасслоением.

3.1.10. Лемма. Пусть задана коммутативная диаграмма

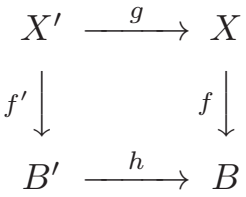

в sSp, где g и $h$ - слабые эквивалентности, a $f$ u $f^{\prime}$ - квазирасслоения. Квазирасслоение $f$ является левым тогда и только тогда, когда левым является $f^{\prime}$. 
Доказательство. Рассмотрим коммутативную диаграмму, индуцированную диаграммой (3.1):

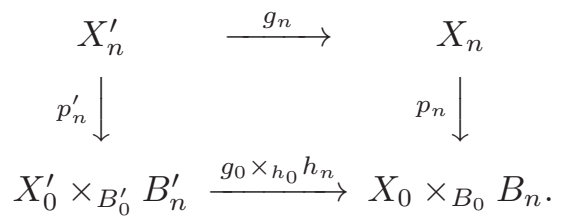

Нам нужно доказать, что $p_{n}$ является слабой эквивалентностью тогда и только тогда, когда слабой эквивалентностью является $p_{n}^{\prime}$. Поскольку $g$ - слабая эквивалентность, достаточно показать, что $g_{0} \times_{h_{0}} h_{n}$-слабая эквивалентность.

Согласно лемме 3.1.7, отображения $X_{0} \rightarrow B_{0}$ и $X_{0}^{\prime} \rightarrow B_{0}^{\prime}$ являются квазирасслоениями. Поэтому $g_{0} \times_{h_{0}} h_{n}$ - слабая эквивалентность по предположению и в силу следствия 3.1.5.

3.1.11. Следствие. Фибрантная замена левого квазирасслоения есть левое расслоение.

Доказательство. Применим лемму 3.1 .10 и замечание 3.1.9 в случае, когда $B^{\prime}=B$, а $f$ - фибрантная замена квазирасслоения $f^{\prime}$.

\section{2. Продолжение расслоений и фибрантные замены.}

3.2.1. Лемма. Пусть задана коммутативная диаграмма

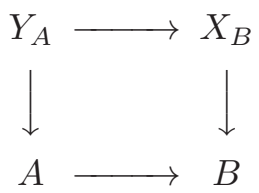

в sSp, такая, что вертикальные стрелки-расслоения, горизонтальные стрелки - корасслоения, а индуцированное отображение $Y_{A} \rightarrow X_{A}:=X_{B} \times_{B} A$ - тривиальное корасслоение, и пусть $Y_{B}$ - наибольшее симплициальное подпространство в $X_{B}$, такое, что $Y_{B} \times_{B} A=Y_{A}$.

Тогда $Y_{B} \subset X_{B}-$ сильный деформаиионный ретракт над $B$. В частности, $Y_{B} \rightarrow B$ - расслоение, а включение $i: Y_{B} \hookrightarrow X_{B}$ - тривиальное корасслоение.

Доказательство. Рассмотрим симплициальное подпространство $Y_{B} \subset X_{B}$, у которого $\left(Y_{B}\right)_{n, m}$ - множество всех $\tau \in\left(X_{B}\right)_{n, m}=\operatorname{Hom}\left(\square[n, m], X_{B}\right)$, таких, что $\tau\left(\tau^{-1}\left(X_{A}\right)\right) \subset Y_{A}$. Тогда $Y_{B} \subset X_{B}$-наибольшее подпространство, такое, что $Y_{B} \times_{B} A=Y_{A}$. По построению для любого морфизма $C \rightarrow B$ множество $\operatorname{Hom}_{B}\left(C, Y_{B}\right)$ можно отождествить с множеством отображений $f \in$ $\operatorname{Hom}_{B}\left(C, X_{B}\right)$, таких, что $f\left(C \times_{B} A\right) \subset Y_{A}$.

Так как $Y_{A} \hookrightarrow X_{A}$ - тривиальное корасслоение расслоений над $A$, то $Y_{A} \subset$ $X_{A}$ - сильный деформационный ретракт над $A$ (см. 1.3.10(c)). Таким образом, существует отображение $g: X_{A} \times \Delta[1] \rightarrow X_{A}$ над $A$, такое, что (i) $\left.g\right|_{X_{A} \times\{0\}}=$ $\operatorname{Id}_{X_{A}}$, (ii) $g\left(X_{A} \times\{1\}\right) \subset Y_{A}$ и (iii) $\left.g\right|_{Y_{A} \times \Delta[1]}=\operatorname{pr}_{1}: Y_{A} \times \Delta[1] \rightarrow Y_{A} \subset X_{A}$.

Поскольку $Y_{B} \cap X_{A}=Y_{A}$, из свойства (iii) отображения $g$ следует, что оно расширяется до отображения $g^{\prime}:\left(X_{A} \sqcup_{Y_{A}} Y_{B}\right) \times \Delta[1] \rightarrow X_{B}$ над $B$, такого, что $\left.g^{\prime}\right|_{Y_{B} \times \Delta[1]}=\operatorname{pr}_{1}$. Далее, из свойства (i) отображения $g$ следует, что $g^{\prime}$ расширяется до морфизма

$$
g^{\prime \prime}:\left(\left(X_{A} \sqcup_{Y_{A}} Y_{B}\right) \times \Delta[1]\right) \sqcup_{\left(X_{A} \sqcup_{Y_{A}} Y_{B}\right) \times\{0\}}\left(X_{B} \times\{0\}\right) \rightarrow X_{B}
$$


над $B$, такого, что $\left.g^{\prime \prime}\right|_{X_{B} \times\{0\}}=\operatorname{Id}_{X_{B}}$.

Поскольку $\{0\} \hookrightarrow \Delta[1]$ - тривиальное корасслоение, а модельная категория $s S p$ декартова, заключаем, что

$$
\left(\left(X_{A} \sqcup_{Y_{A}} Y_{B}\right) \times \Delta[1]\right) \sqcup_{\left(X_{A} \sqcup_{Y_{A}} Y_{B}\right) \times\{0\}}\left(X_{B} \times\{0\}\right) \hookrightarrow X_{B} \times \Delta[1]
$$

- тривиальное корасслоение, так что $g^{\prime \prime}$ расширяется до отображения $h: X_{B} \times$ $\Delta[1] \rightarrow X_{B}$ над $B$.

Тогда $h$ удовлетворяет соотношениям $\left.h\right|_{X_{B} \times\{0\}}=\left.g^{\prime \prime}\right|_{X_{B} \times\{0\}}=\operatorname{Id}_{X_{B}},\left.h\right|_{Y_{B} \times \Delta[1]}$ $=\left.g^{\prime}\right|_{Y_{B} \times \Delta[1]}=\operatorname{pr}_{1}$ и $h\left(X_{A} \times\{1\}\right)=g\left(X_{A} \times\{1\}\right) \subset Y_{A}$. Таким образом, в силу конструкции пространства $Y_{B}$ получаем, что $h\left(X_{B} \times\{1\}\right) \subset Y_{B}$. Иными словами, $h$ реализует $Y_{B} \subset X_{B}$ как сильный деформационный ретракт над $B$. Последнее утверждение следует из замечания 1.3.10(b).

3.2.2. Следствие. Пусть $f: X \rightarrow B-$ квазирасслоение, и пусть $A \hookrightarrow B-$ корасслоение, такое, что $X \times_{B} A \rightarrow A$ является расслоением. Тогда существует фибрантная замена $f^{\prime}: X^{\prime} \rightarrow B$ квазирасслоения $f$, такая, что $X \times_{B} A=$ $X^{\prime} \times_{B} A$.

Доказательство. Пусть $X \stackrel{i}{\longrightarrow} Y^{\prime} \stackrel{p}{\longrightarrow} B$ - произвольное разложение квазирасслоения $f$, где $i$ - тривиальное корасслоение, а $p$-расслоение. Поскольку $f$-квазирасслоение, $i$ индуцирует тривиальное корасслоение $X \times_{B} A \hookrightarrow$ $Y^{\prime} \times_{B} A$ (по лемме 3.1.3(b)).

Пусть $X^{\prime} \subset Y^{\prime}$ - наибольшее симплициальное подпространство, такое, что $X^{\prime} \times_{B} A=X \times_{B} A$. Тогда $f^{\prime}: X^{\prime} \rightarrow B$ - расслоение, а $X^{\prime} \hookrightarrow Y^{\prime}$ - тривиальное корасслоение (по лемме 3.2.1). Так как тривиальное корасслоение $i$ разлагается в композицию $X \stackrel{j}{\longrightarrow} X^{\prime} \hookrightarrow Y^{\prime}$, то отображение $j$ есть тривиальное корасслоение в силу свойства $\ll 2$ из $3 »$, так что $f^{\prime}$ - фибрантная замена квазирасслоения $f$.

3.2.3. Доказательство леммы 1.3.11(d). Композиция $Y_{A} \stackrel{f}{\longrightarrow} A \rightarrow B$ разлагается в композицию тривиального корасслоения $i: Y_{A} \rightarrow X_{B}$ и расслоения $p: X_{B} \rightarrow B$. Положим $X_{A}:=X_{B} \times_{B} A$. Тогда $i$ разлагается в композицию $Y_{A} \stackrel{i^{\prime}}{\longrightarrow} X_{A} \stackrel{i^{\prime \prime}}{\longrightarrow} X_{B}$. Кроме того, $i^{\prime \prime}$ - слабая эквивалентность, потому что $A \rightarrow B$ - слабая эквивалентность, $p$ - расслоение, а модельная структура Риди правильна справа, так что $i^{\prime}$ - слабая эквивалентность в силу свойства «2 из $3 »$. Пусть теперь $Y_{B} \subset X_{B}$ - наибольшее симплициальное подпространство, такое, что $Y_{B} \times_{B} A=Y_{A}$. Тогда $g: Y_{B} \rightarrow B$ - расслоение по лемме 3.2.1, и его сужение на $A$ есть $f$.

3.2.4. Обозначения. (а) Для $X \in s S p$ и $i \geqslant 0$ определим $i$-остов $s k_{i} X$ как наименьшее симплициальное подпространство $Y \subset X$, такое, что $Y_{n, m}=X_{n, m}$ для всех $n+m \leqslant i$. Тогда $\emptyset=s k_{-1} X \subset s k_{0} X \subset \cdots \subset s k_{i} X \subset \cdots \subset X$ и $X=\operatorname{colim}_{i} s k_{i} X$.

(b) Для любых $n, m \geqslant 0$ обозначим через $X_{n, m}^{n d}:=X_{n, m} \backslash\left(s k_{n+m-1} X\right)_{n, m}$ множество «невырожденных бисимплексов». Тогда $X_{0,0}^{n d}=X_{0,0}$ и для любого $i>0 \quad i$-остов $s k_{i} X$ естественно изоморфен пушауту $(i-1)$-остова $s k_{i-1} X$ и $\bigsqcup_{n+m=i, a \in X_{n, m}^{n d}} \square[n, m]$ над $\bigsqcup_{n+m=i, a \in X_{n, m}^{n d}} \partial \square[n, m]$.

3.2.5. Лемма. Пусть $f: X \rightarrow B-$ морфизм в $(s S p / B)_{\mathscr{U}}$, где $B \in s S p|\mathscr{U}|$. Тогда у $f$ есть фибрантная замена $f^{\prime}: X^{\prime} \rightarrow B$ в $(s S p / B)_{\mathscr{U}}$. 
Доказательство. Поскольку $(s S p / B)_{\mathscr{U}} \rightarrow(s S p / B)_{|\mathscr{U}|}$ - эквивалентность категорий (см. 2.2.2(d)), достаточно доказать существование фибрантной замены $f^{\prime}$ в $(s S p / B)_{|\mathscr{U}|}$. Так как $B \in s S p_{|\mathscr{U}|}$ и $|\mathscr{U} \times \mathscr{U}|=|\mathscr{U}|$, то $X \in s S p_{|\mathscr{U}|}$. Кроме того, опять пользуясь равенством $|\mathscr{U} \times \mathscr{U}|=|\mathscr{U}|$, получаем, что фибрантная замена $f^{\prime}: X^{\prime} \rightarrow B$, построенная с помощью «аргумента малых объектов» (см., например, $[9$, II.3, лемма 3 и следующее за ней замечание]), удовлетворяет соотношению $X^{\prime} \in s S p_{|\mathscr{U}|}$, так что $f^{\prime} \in(s S p / B)_{|\mathscr{U}|}$.

3.2.6. Следствие. $B$ ситуации следствия 3.2 .2 предположим, ито $f: X \rightarrow$ $B$ - квазирасслоение в $(s S p / B)_{\mathscr{U}}$. Тогда $f^{\prime}: X^{\prime} \rightarrow B$ также можно выбрать в $(s S p / B) \mathscr{U}$.

Доказательство. (I) Предположим сначала, что $B \in s S p|\mathscr{U}|$. Тогда по лемме 3.2.5 расслоение $Y^{\prime} \rightarrow B$ в доказательстве следствия 3.2.2 можно выбрать из $(s S p / B)_{\mathscr{U}}$, а в этом случае $f^{\prime}: X^{\prime} \rightarrow B$ также лежит в $(s S p / B)_{\mathscr{U}}$.

(II) Для каждого $i$ положим $B^{[i]}:=s k_{i} B \cup A \subset B$. Мы утверждаем, что для любого квазирасслоения $g: Y \rightarrow B^{[i]}$ из $\left(s S p / B^{[i]}\right)_{\mathscr{U}}$, сужение которого на $B^{[i-1]}$ есть расслоение, существует фибрантная замена $g^{\prime}$ из $\left(s S p / B^{[i]}\right)_{\mathscr{U}}$, такая, что $\left.g^{\prime}\right|_{B^{[i-1]}}=\left.g\right|_{B^{[i-1]}}$.

Согласно 3.2.4(b), имеем равенство $B^{[i]}=B^{[i-1]} \sqcup_{\left(\sqcup_{a} \partial \square[n, m]\right)}\left(\bigsqcup_{a} \square[n, m]\right)$, где $a$ пробегает множество всех отображений $\square[n, m] \rightarrow B^{[i]}, n+m=i$, образ которых не лежит в $B^{[i-1]}$. Для каждого такого $a$ положим $g_{a}:=a^{*}(g)$. Тогда $g_{a}: Y_{a} \rightarrow \square[n, m]$ - квазирасслоение из $(s S p / \square[n, m])_{\mathscr{U}}$, сужение которого на $\partial \square[n, m]$ есть расслоение. Таким образом, в силу (I) существует фибрантная замена $g_{a}^{\prime}: Y_{a}^{\prime} \rightarrow \square[n, m]$ квазирасслоения $g_{a}$, принадлежащая $(s S p / \square[n, m])_{\mathscr{U}}$, такая, что $\left.g_{a}^{\prime}\right|_{\partial \square[n, m]}=\left.g_{a}\right|_{\partial \square[n, m]}$.

Положим $Y^{\prime}:=Y \sqcup_{\left(\sqcup_{a} Y_{a}\right)}\left(\bigsqcup_{a} Y_{a}^{\prime}\right)$ и $g^{\prime}:=g \sqcup_{\left(\sqcup_{a} g_{a}\right)}\left(\bigsqcup_{a} g_{a}^{\prime}\right): Y^{\prime} \rightarrow B^{[i]}$. Тогда $g^{\prime}$ - расслоение, потому что его сужение на каждое $\square[n, m]$ есть расслоение (мы воспользовались 1.3.7(а)). Кроме того, каноническое отображение $Y \rightarrow Y^{\prime}$ есть пушаут несвязного объединения тривиальных корасслоений $Y_{a} \hookrightarrow Y_{a}^{\prime}$. Таким образом, оно является тривиальным корасслоением, ибо категория $s S p$ правильна слева.

(III) Сначала построим последовательность тривиальных корасслоений $X=$ $X^{\prime[-1]} \hookrightarrow X^{\prime[0]} \hookrightarrow X^{\prime[1]} \hookrightarrow \cdots$ над $B$, таких, что $f^{\prime[i]}: X^{\prime[i]} \rightarrow B-$ квазирасслоение из $(s S p / B)_{\mathscr{U}},\left.f^{\prime[i]}\right|_{B^{[i]}}:\left.X^{\prime[i]}\right|_{B^{[i]}} \rightarrow B^{[i]}-$ расслоение и $\left.X^{\prime[i+1]}\right|_{B^{[i]}}=\left.X^{\prime[i]}\right|_{B^{[i]}}$ для всех $i$.

Рассуждая по индукции, предположим, что $X^{\prime[i-1]}$ уже построено. Тогда отображение $g:=\left.f^{\prime[i-1]}\right|_{B^{[i]}}$ есть квазирасслоение из $\left(s S p / B^{[i]}\right)_{\mathscr{U}}$, сужение которого на $B^{[i-1]}$ является расслоением. Таким образом, в силу (II) существует его фибрантная замена $g^{\prime}: Y^{\prime} \rightarrow B^{[i]}$ из $\left(s S p / B^{[i]}\right)_{\mathscr{U}}$, такая, что $\left.g^{\prime}\right|_{B^{[i-1]}}=\left.g\right|_{B^{[i-1]}}$. Положим $X^{\prime[i]}:=X^{\prime[i-1]} \sqcup_{\left.X^{\prime[i-1]}\right|_{B}[i]} Y^{\prime}$.

Из леммы 3.1.6 следует, что $f^{\prime[i]}: X^{\prime[i]} \rightarrow B$ - квазирасслоение и что $X^{\prime[i-1]} \rightarrow$ $X^{\prime[i]}$ - слабая эквивалентность, в то время как по построению $f^{\prime[i]}$ принадлежит $(s S p / B)_{\mathscr{U}},\left.f^{\prime[i]}\right|_{B^{[i]}}=g^{\prime}$ - расслоение и $\left.X^{\prime[i]}\right|_{B^{[i-1]}}=\left.X^{\prime[i-1]}\right|_{B^{[i-1]}}$.

Наконец, положим $f^{\prime}:=\operatorname{colim}_{i} f^{\prime[i]}: X^{\prime} \rightarrow B$. Тогда $f^{\prime}$ удовлетворяет соотношению $\left.f^{\prime}\right|_{B^{[i]}}=\left.f^{\prime[i]}\right|_{B^{[i]}}$, так что $f^{\prime}$ - расслоение и $f^{\prime} \in(s S p / B)_{\mathscr{U}}$. Кроме того, так как $X^{\prime[i]} \hookrightarrow X^{\prime[i+1]}$ - тривиальное корасслоение для каждого $i$, то 
$X \hookrightarrow X^{\prime}$ - также тривиальное корасслоение, так что $f^{\prime}$ есть фибрантная замена квазирасслоения $f$.

\section{§4. Дополнения}

\section{1. Дискретный итерированный цилиндр.}

4.1.1. Наблюдения. (а) Каждое отображение $p: X \rightarrow B \times F[m]$ в $s S p$ индуцирует отображение $p_{n}: X_{n} \rightarrow B_{n} \times F[m]_{n}$ для всех $n$. Так как $F[m]_{n}$ имеет разложение $\bigsqcup_{\tau:[n] \rightarrow[m]} \mathrm{pt}$, то каждое $X_{n}$ разлагается в несвязное объединение $X_{n}=\bigsqcup_{\tau:[n] \rightarrow[m]} X_{\tau}$, а $p_{n}$ разлагается в несвязное объединение отображений $p_{\tau}: X_{\tau} \rightarrow B_{n}$.

(b) По определению все слои отображения $p$ принадлежат Set $\mathscr{\ell}$ тогда и только тогда, когда все слои каждого $p_{\tau}$ принадлежат Setथ .

(c) Заметим, что $p_{n}$ является квазирасслоением тогда и только тогда, когда каждое $p_{\tau}: X_{\tau} \rightarrow B_{n}$ - квазирасслоение. Используя лемму 3.1.7, заключаем, что $p$ является квазирасслоением тогда и только тогда, когда каждое $p_{\tau}-$ квазирасслоение.

(d) Напомним, что квазирасслоение $p: X \rightarrow B \times F[m]$ является левым тогда и только тогда, когда каждый морфизм $g_{n}: X_{n} \rightarrow X_{0} \times(B \times F[m])_{0}(B \times F[m])_{n}$ есть слабая эквивалентность. Заметим, что $g_{n}$ разлагается в несвязное объединение морфизмов $g_{\tau}: X_{\tau} \rightarrow X_{\left.\tau\right|_{0}} \times_{B_{0}} B_{n}$. Таким образом, $f$ является левым тогда и только тогда, когда каждое $g_{\tau}$ - слабая эквивалентность.

(е) Морфизм $f: X \rightarrow Y$ левых квазирасслоений над $B \times F[m]$ является слабой эквивалентностью тогда и только тогда, когда $\left.f\right|_{i}:\left.\left.X\right|_{i} \rightarrow Y\right|_{i}$ - слабая эквивалентность для любого $i=0, \ldots, m$. Действительно, согласно рассуждению из леммы 2.1.5, $f$ является слабой эквивалентностью тогда и только тогда, когда $f_{0}$ - слабая эквивалентность. Поскольку $F[n]_{0}=\bigsqcup_{i=0}^{n} \delta^{i}(\mathrm{pt})$, отсюда следует нужное утверждение.

4.1.2. Дискретный итерированный цилиндр. Для $B \in s S p$ и последовательности морфизмов $f: K^{(0)} \stackrel{f_{1}}{\longrightarrow} \cdots \stackrel{f_{m}}{\longrightarrow} K^{(m)}$ из $s S p / B$ рекуррентно определим дискретный итерированный иилиндр $\mathrm{Cyl}^{d}(f) \rightarrow B \times F[m]$ и морфизм $\iota_{j}: K^{(j)} \times e^{j} F[m-j] \rightarrow \mathrm{Cyl}^{d}(f)$ над $B \times F[m]$ для всех $j=0, \ldots, m$ следующим образом.

Для $m=0$ положим $\mathrm{Cyl}^{d}(f):=K^{(0)}$ и $\iota_{0}=\mathrm{Id}$. Для $m \geqslant 1$ обозначим через $f(1)$ последовательность $f(1): K^{(1)} \stackrel{f_{2}}{\longrightarrow} \cdots \stackrel{f_{m}}{\longrightarrow} K^{(m)}$ и предположим по индукции, что мы уже определили итерированный цилиндр $\mathrm{Cyl}^{d}(f(1)) \rightarrow B \times$ $F[m-1]$ и морфизм $\iota_{j}: K^{(j)} \times e^{j} F[m-j] \rightarrow e^{1} \mathrm{Cyl}^{d}(f(1))$ для всех $j=1, \ldots, m$.

Определим $\mathrm{Cyl}^{d}(f) \rightarrow B \times F[m]$ как пушаут

$$
\mathrm{Cyl}^{d}(f):=\left(K^{(0)} \times F[m]\right) \sqcup_{\left(K^{(0)} \times e^{1} F[m-1]\right)} e^{1} \mathrm{Cyl}^{d}(f(1)),
$$

где отображение $K^{(0)} \times e^{1} F[m-1] \rightarrow e^{1} \mathrm{Cyl}^{d}(f(1))$ определено как композиция

$$
K^{(0)} \times e^{1} F[m-1] \stackrel{f_{1}}{\longrightarrow} K^{(1)} \times e^{1} F[m-1] \stackrel{\iota_{1}}{\longrightarrow} e^{1} \mathrm{Cyl}^{d}(f(1)) .
$$

Наконец, определим $\iota_{0}: K^{(0)} \times F[m] \rightarrow \mathrm{Cyl}^{d}(f)$ как естественное отображение. 
4.1.3. Лемма. Если каждое отображсение $K^{(i)} \rightarrow B-($ левое) квазирас-

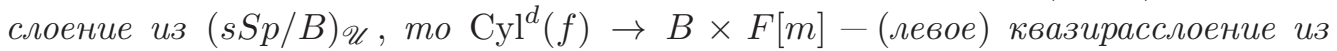
$(s S p / B \times F[m]) \mathscr{U}$.

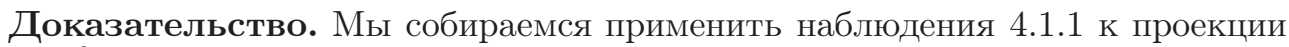
$p: \mathrm{Cyl}^{d}(f) \rightarrow B \times F[m]$.

Мы утверждаем, что $\mathrm{Cyl}^{d}(f)_{\tau}=K_{n}^{(\tau(0))}$ для любого $\tau:[n] \rightarrow[m]$. Доказательство проводится по индукции. Утверждение очевидно при $m=0$. Далее, если $m \geqslant 1$, то из (4.1) следует, что $\mathrm{Cyl}^{d}(f)_{\tau}$ равно $K_{n}^{(0)}$, если $\tau(0)=0$, и равно $\mathrm{Cyl}^{d}(f(1))_{\tau^{\prime}}$, где $\tau^{\prime}:[n] \rightarrow[m-1]$ дается формулой $\tau^{\prime}(i)=\tau(i)-1$, если $\tau(0) \geqslant 1$. По предположению индукции во втором случае $\mathrm{Cyl}^{d}(f)_{\tau}$ равно $K_{n}^{\left(\tau^{\prime}(0)+1\right)}=K_{n}^{(\tau(0))}$.

Согласно доказанному выше и 4.1.1(a), каждый слой отображения $p$ является слоем некоторого $K_{n}^{(\tau(0))} \rightarrow B_{n}$. Таким образом, он принадлежит Setש, потому что $K^{(\tau(0))} \rightarrow B$ лежит в $(s S p / B) \mathscr{U}$.

Кроме того, так как каждое $K^{(\tau(0))} \rightarrow B$ - квазирасслоение, то и каждое $K_{n}^{(\tau(0))} \rightarrow B_{n}$ - квазирасслоение (по лемме 3.1 .7$)$. Поэтому $p$ - квазирасслоение в силу 4.1.1(c). Аналогично каждая проекция $\mathrm{Cyl}^{d}(f)_{\tau} \rightarrow \mathrm{Cyl}^{d}(f)_{\left.\tau\right|_{0}} \times_{B_{0}} B_{n}-$ это просто $K_{n}^{(\tau(0))} \rightarrow K_{0}^{(\tau(0))} \times B_{0} B_{n}$. Поэтому она является слабой эквивалентностью, ибо расслоение $K^{(\tau(0))} \rightarrow B$ левое. Теперь нужное утверждение следует из $4.1 .1(\mathrm{~d})$.

4.1.4. Лемма. Пусть $f: X \rightarrow Y$-отображсение, $a E \rightarrow Y \times F[n]$ - левое расслоение.

(a) Отображсение $p: \mathscr{M} \operatorname{pp}_{Y \times F[n]}(X \times F[n], E) \rightarrow \mathscr{M} a p_{Y \times F[n]}(X, E)$, индуиированное включением $e^{0}: F[0] \hookrightarrow F[n],-$ тривиальное расслоение.

(b) Отображение

$$
q: \mathscr{M} a p_{Y \times F[n]}(X \times F[n], E) \rightarrow \mathscr{M} a p_{Y \times F[n]}\left(X \times\left(e^{0} F[1] \sqcup_{e^{1} F[0]} e^{1} F[n-1]\right), E\right),
$$

индуиированное включением $e^{0} F[1] \sqcup_{e^{1} F[0]} e^{1} F[n-1] \hookrightarrow F[n],-$ тривиальное расслоение.

Доказательство. (а) Так как $E \rightarrow Y \times F[n]$ - левое расслоение, то отображение $E^{X} \rightarrow(Y \times F[n])^{X}$ - левое расслоение по лемме 2.1.2(b). Тогда по лемме 2.1.3(b) отображение

$$
E^{X \times F[n]} \rightarrow E^{X} \times(Y \times F[n])^{X}(Y \times F[n])^{X \times F[n]}
$$

— тривиальное расслоение. Взяв слой над $f \times \operatorname{Id}_{F[n]} \in(Y \times F[n])^{X \times F[n]}$, получаем нужное утверждение.

(b) Так как $e^{0} F[1] \sqcup_{e^{1} F[0]} e^{1} F[n-1] \hookrightarrow F[n]$ - корасслоение, то отображение $q$ - расслоение. Таким образом, остается показать, что $q$-слабая эквивалентность. Рассмотрим отображение

$$
r: \mathscr{M} a p_{Y \times F[n]}\left(X \times\left(e^{0} F[1] \sqcup_{e^{1} F[0]} e^{1} F[n-1]\right), E\right) \rightarrow \mathscr{M} a p_{Y \times F[n]}(X, E),
$$

индуцированное включением $F[0] \stackrel{e^{0}}{\longrightarrow} e^{0} F[1] \hookrightarrow e^{0} F[1] \sqcup_{e^{1} F[0]} e^{1} F[n-1]$. Поскольку $r \circ q=p$, оно является слабой эквивалентностью в силу (а). Таким 
образом, остается показать, что $r$ - слабая эквивалентность. Но $r$ можно записать как композицию отображений

$$
\mathscr{M} a p_{Y \times F[n]}\left(X \times\left(e^{0} F[1] \sqcup_{e^{1} F[0]} e^{1} F[n-1]\right), E\right) \rightarrow \mathscr{M} a p_{Y \times F[n]}\left(X \times e^{0} F[1], E\right)
$$

и $\left(e^{0}\right)^{*}: \mathscr{M}_{a p_{Y \times F[n]}}\left(X \times e^{0} F[1], E\right) \rightarrow \mathscr{M} a p_{Y \times F[n]}(X, E)$, так что остается показать, что оба отображения - тривиальные расслоения. Поскольку первое отображение - пулбэк морфизма $\mathscr{M}_{a p_{Y \times F[n]}}\left(X \times e^{1} F[n-1], E\right) \rightarrow \mathscr{M} a p_{Y \times F[n]}(X \times$ $\left.e^{1} F[0], E\right)$, оба отображения являются тривиальными расслоениями в силу (а).

4.2. Доказательства. В этом разделе мы докажем предложения 2.2.8, 2.2.9 и следствие 2.2.10. Самой трудной частью является предложение 2.2.9(b), доказательство которого проводится в 4.2.7-4.2.9 и 4.2.11-4.2.13. Обозначим $\mathfrak{S}_{\mathscr{U}}$ просто через $\mathfrak{S}$.

4.2.1. Доказательство предложения 2.2.8(a). Согласно 1.3.7(a), нужно доказать, что для любого тривиального корасслоения $i: A \rightarrow B$ из $s S p$, где $B=\square[n, m]$, морфизм $i^{*}: \operatorname{Hom}(B, \mathfrak{S}) \rightarrow \operatorname{Hom}(A, \mathfrak{S})$ сюръективен. Согласно лемме 2.2.4, это означает, что каждое левое расслоение $Y_{A} \rightarrow A$ в $(s S p / A)_{\mathscr{U}}$ расширяется до левого расслоения $Y_{B} \rightarrow B$ в $(s S p / B)_{\mathscr{U}}$.

Из леммы 1.3.11(d) (см. 3.2.3) следует, что существует расслоение $Y_{B} \rightarrow B$, продолжающее $Y_{A} \rightarrow A$. Кроме того, поскольку композиция $Y_{A} \rightarrow A \rightarrow B$ принадлежит $(s S p / B)_{\mathscr{U}}$ и $B \in s S p_{|\mathscr{U}|}$, расслоение $X_{B} \rightarrow B$ в 3.2 .3 можно выбрать из $(s S p / B)_{\mathscr{U}}$ (по лемме 3.2 .5 ), а в этом случае $Y_{B} \rightarrow B$ также принадлежит $(s S p / B)_{\mathscr{U}}$.

Так как $A \rightarrow B$ - слабая эквивалентность и модельная структура Риди правильна, то включение $Y_{A} \rightarrow Y_{B}$ - слабая эквивалентность. Поскольку $Y_{A} \rightarrow A$ - левое расслоение, $Y_{B} \rightarrow B$-левое расслоение по лемме 3.1.10.

4.2.2. Доказательство предложения 2.2.8(b). Чтобы показать, что $\mathfrak{S}^{(n)} \rightarrow \mathfrak{S}^{n+1}-$ расслоение, нужно доказать, что для любого тривиального корасслоения $A \hookrightarrow B$ отображение $\left(\mathfrak{S}^{(n)}\right)^{B} \rightarrow\left(\mathfrak{S}^{n+1}\right)^{B} \times\left(\mathfrak{S}^{n+1}\right)^{A}\left(\mathfrak{S}^{(n)}\right)^{A}$ сюръективно. Используя сделанное в 2.2 .7 наблюдение, нужно доказать, что для любого упорядоченного набора из $n+1$ левых расслоений $E^{(0)}, \ldots, E^{(n)}$ над $B$ каждая диаграмма $\left.\left.E^{(0)}\right|_{A} \rightarrow \cdots \rightarrow E^{(n)}\right|_{A}$ над $A$ расширяется до диаграммы $E^{(0)} \rightarrow \cdots \rightarrow E^{(n)}$ над $B$.

Для этого достаточно показать, что каждое отображение сужения

$$
\operatorname{Hom}_{B}\left(E^{(i)}, E^{(i+1)}\right) \rightarrow \operatorname{Hom}_{B}\left(\left.E^{(i)}\right|_{A}, E^{(i+1)}\right)=\operatorname{Hom}_{A}\left(\left.E^{(i)}\right|_{A},\left.E^{(i+1)}\right|_{A}\right)
$$

сюръективно. Так как $E^{(i)} \rightarrow B$-расслоение, $A \rightarrow B$ - тривиальное корасслоение и модельная структура Риди правильна, то $\left.E^{(i)}\right|_{A} \rightarrow E^{(i)}$ - тривиальное корасслоение. Таким образом, утверждение следует из того факта, что $E^{(i+1)} \rightarrow B$ - расслоение.

Чтобы показать, что $\mathfrak{S}^{(w e)} \rightarrow \mathfrak{S}^{2}-$ расслоение, мы рассуждаем дословно, как и выше, и замечаем, что поскольку корасслоения $\left.E^{(i)}\right|_{A} \rightarrow E^{(i)}$ тривиальны, из свойства «2 из $3 »$ следует, что морфизм $E^{(0)} \rightarrow E^{(1)}$ есть слабая эквивалентность тогда и только тогда, когда его сужение $\left.\left.E^{(0)}\right|_{A} \rightarrow E^{(1)}\right|_{A}-$ слабая эквивалентность. 
4.2.3. Доказательство предложения 2.2.8(c). Нам нужно доказать, что для любого корасслоения $A \hookrightarrow B$ отображение $\left(\mathfrak{S}^{(w e)}\right)^{B} \rightarrow \mathfrak{S}^{B} \times \mathfrak{S}^{A}\left(\mathfrak{S}^{(w e)}\right)^{A}$ сюръективно. Пусть $E^{(0)} \rightarrow B$ принадлежит $(L F i b / B)_{\mathscr{U}}, E_{A}^{(1)} \rightarrow A$ принадлежит $(L F i b / A)_{\mathscr{U}}$, а $\phi^{\prime}:\left.E^{(0)}\right|_{A} \rightarrow E_{A}^{(1)}$ - слабая эквивалентность над $A$. Нам нужно доказать, что $\phi^{\prime}$ расширяется до слабой эквивалентности $\phi: E^{(0)} \rightarrow E^{(1)}$ над $B$, такой, что $E^{(1)} \rightarrow B$ принадлежит $(L F i b / B)_{\mathscr{U}}$.

Согласно лемме 3.1.6, пушаут $E^{(0)} \sqcup_{\left.E^{(0)}\right|_{A}} E_{A}^{(1)}$ - квазирасслоение над $B$, сужение которого на $A$-это расслоение $E_{A}^{(1)} \rightarrow A$. Поэтому в силу следствия 3.2.6 существует его фибрантная замена $E^{(1)} \rightarrow B$ в $(s S p / B)_{\mathscr{U}}$, такая, что $\left.E^{(1)}\right|_{A}=E_{A}^{(1)}$.

По построению $\phi^{\prime}$ расширяется до морфизма $\phi: E^{(0)} \stackrel{\phi_{1}}{\longrightarrow} E^{(0)} \sqcup_{\left.E^{(0)}\right|_{A}} E_{A}^{(1)}$ $\stackrel{\phi_{2}}{\longrightarrow} E^{(1)}$ расслоений над $B$. Кроме того, так как $\phi^{\prime}$ - слабая эквивалентность, то ее пушаут $\phi_{1}$ - также слабая эквивалентность, так что и $\phi$ - тоже слабая эквивалентность. Поскольку $E^{(0)} \rightarrow B$-левое расслоение, $E^{(1)} \rightarrow B$-левое расслоение по лемме 3.1.10.

4.2.4. Доказательство предложения 2.2.8(d). Используя наблюдения, сделанные в 2.2.7(b), остается показать, что если $a \in \mathfrak{S}^{(w e)}, b \in \mathfrak{S}^{(1)}$, причем $a \sim b$ в $\mathfrak{S}^{(1)}$, то $b \in \mathfrak{S}^{(w e)}$. Поскольку $\mathfrak{S}^{(w e)} \rightarrow \mathfrak{S} \times \mathfrak{S}-$ расслоение, в то время как $\mathfrak{S}^{(1)}$ фибрантно (в силу предложения 2.2.8(a),(b)), можно изменить $а$ таким образом, что $a \sim b$ в некотором слое расслоения $\mathfrak{S}^{(1)} \rightarrow \mathfrak{S} \times \mathfrak{S}$.

Тогда элементу а соответствует слабая эквивалентность $\phi_{a}: E^{(0)} \rightarrow E^{(1)}$, элементу $b$ соответствует морфизм $\phi_{b}: E^{(0)} \rightarrow E^{(1)}$, а предположение, что $a \sim b$, означает, что отображения $\phi_{a}$ и $\phi_{b}$ гомотопны. Поэтому $\phi_{b}$ - слабая эквивалентность (в силу 1.3.9(c)), так что $b \in \mathfrak{S}^{(w e)}$.

4.2.5. Доказательство предложения 2.2.9(a). Согласно лемме 2.2.4, для любого $K \in s S p$ множество $\operatorname{Hom}\left(K, \mathfrak{S}^{\Delta[1]}\right)=\operatorname{Hom}(K \times \Delta[1], \mathfrak{S})$ находится во взаимно однозначном соответствии с множеством левых расслоений $E \rightarrow$ $K \times \Delta[1]$ в $(s S p / K \times \Delta[1])_{\mathscr{U}}$, в то время как множество $\operatorname{Hom}\left(K, \mathfrak{S}^{2}\right)$ находится во взаимно однозначном соответствии с множеством пар левых расслоений $E^{(0)} \rightarrow K, E^{(1)} \rightarrow K$ из $(s S p / K) \mathscr{U}$. Кроме того, проекция $\mathfrak{S}^{(w e)} \rightarrow \mathfrak{S}^{2}$ переводит $E \rightarrow K \times \Delta[1]$ в пару $\left.E\right|_{0}:=\left.E\right|_{K \times\{0\}},\left.E\right|_{1}:=\left.E\right|_{K \times\{1\}}$, а множество $\operatorname{Hom}\left(K, \mathfrak{S}^{(w e)}\right)$ находится во взаимно однозначном соответствии с множеством слабых эквивалентностей $\phi: E^{(0)} \rightarrow E^{(1)}$ левых расслоений над $K$ (в силу 2.2.7).

Согласно лемме 1.3.11(c), для любого левого расслоения $E \rightarrow K \times \Delta[1]$ существует слабая эквивалентность $\phi:\left.\left.E\right|_{0} \rightarrow E\right|_{1}$ левых расслоений над $K$. Если $K:=\mathfrak{S}^{\Delta[1]}$ и $E$ - левое расслоение, соответствующее $\operatorname{Id}_{K}$, то отображение $\phi$ индуцирует морфизм $\psi: \mathfrak{S}^{\Delta[1]} \rightarrow \mathfrak{S}^{(\text {we })}$ над $\mathfrak{S}^{2}$.

Кроме того, $p_{0}: \mathfrak{S}^{(w e)} \rightarrow \mathfrak{S}$ - тривиальное расслоение в силу предложения $2.2 .8(\mathrm{c})$, в то время как $\delta_{0}: \mathfrak{S}^{\Delta[1]} \rightarrow \mathfrak{S}-$ тривиальное расслоение, потому что $\mathfrak{S}$ фибрантно (в силу предложение $2.2 .8(\mathrm{a})$ ), а $\delta^{0}: \Delta[0] \rightarrow \Delta[1]$ - тривиальное корасслоение. Тогда $\psi$ - слабая эквивалентность в силу свойства «2 из $3 »$, а значит, и гомотопическая эквивалентность по лемме 1.3.11(b). 
4.2.6. Доказательство следствия 2.2.10. Гомотопическая эквивалентность $\mathfrak{S}^{(w e)} \rightarrow \mathfrak{S}^{\Delta[1]}$ над $\mathfrak{S}^{2}$, установленная в предложении $2.2 .9($ а), индуцирует гомотопическую эквивалентность

$$
\operatorname{Map}\left(K, \mathfrak{S}^{(w e)}\right) \rightarrow \operatorname{Map}\left(K, \mathfrak{S}^{\Delta[1]}\right)=\operatorname{Map}\left(\Delta[1], \mathfrak{S}^{K}\right)
$$

над $\operatorname{Map}\left(K, \mathfrak{S}^{2}\right)$, а значит, и гомотопическую эквивалентность слоев над $(\alpha, \beta) \in$ $\operatorname{Map}\left(K, \mathfrak{S}^{2}\right)$. Поскольку $\operatorname{Map}\left(K, \mathfrak{S}^{(w e)}\right)_{\alpha, \beta} \neq \emptyset$ означает, что $E_{\alpha}$ и $E_{\beta}$ гомотопически эквивалентны над $K\left(\right.$ по 2.2.7(b)), в то время как $\operatorname{Map}\left(\Delta[1], \mathfrak{S}^{K}\right)_{\alpha, \beta} \neq \emptyset$ означает, что $\alpha \sim \beta$ в $\mathfrak{S}^{K}$, получаем нужное утверждение.

4.2.7. Конструкция морфизма $\boldsymbol{\psi}^{(n)}$. Согласно 2.2.7, тождественному отображению $\operatorname{Id}_{\mathfrak{S}^{(n)}}$ соответствует диаграмма $\phi: E^{(0)} \stackrel{\phi_{1}}{\longrightarrow} \cdots \stackrel{\phi_{n}}{\longrightarrow} E^{(n)}$ левых расслоений над $\mathfrak{S}^{(n)}$ в $\left(s S p / \mathfrak{S}^{(n)}\right) \mathscr{U}$. Чтобы определить морфизм $\psi^{(n)}: \mathfrak{S}^{(n)} \rightarrow$ $\mathfrak{S}^{F[n]}$ над $\mathfrak{S}^{n+1}$, нужно построить левое расслоение $E \rightarrow \mathfrak{S}^{(n)} \times F[n]$ в $\left(s S p / \mathfrak{S}^{(n)}\right.$ $\times F[n])_{\mathscr{U}}$, такое, что $\left.E\right|_{i}:=\left.E\right|_{\mathfrak{S}^{(n)} \times\{i\}}=E^{(i)}$.

Рассмотрим дискретный итерированный цилиндр $p: \mathrm{Cyl}^{d}(\phi) \rightarrow \mathfrak{S}^{(n)} \times F[n]$ (см. 4.1.2). Тогда $p$-левое квазирасслоение в $\left(s S p / \mathfrak{S}^{(n)} \times F[n]\right) \mathscr{U}$ (по лемме 4.1.3), и сужение $\left.\mathrm{Cyl}^{d}(\phi)\right|_{i}=E^{(i)}-$ расслоение над $\mathfrak{S}^{(n)}$. Таким образом, в силу следствия 3.2.6 существует фибрантная замена $p^{\prime}: E \rightarrow \mathfrak{S}^{(n)} \times F[n]$ отображения $p$ в $\left(s S p / \mathfrak{S}^{(n)} \times F[n]\right) \mathscr{U}$, такая, что $\left.E\right|_{i}=\left.\mathrm{Cyl}^{d}(\phi)\right|_{i}$ для всех $i$. Тогда $p^{\prime}$ является левым расслоением в силу следствия 3.1.11.

4.2.8. Единственность морфизма $\psi^{(n)}$. Заметим, что если $E^{\prime}$ - другая фибрантная замена для $\mathrm{Cyl}^{d}(\phi) \rightarrow \mathfrak{S}^{(n)} \times F[n]$, такая, что $\left.E^{\prime}\right|_{i}=\left.\mathrm{Cyl}^{d}(\phi)\right|_{i}$ для всех $i$, то существует слабая эквивалентность $E \rightarrow E^{\prime}$ над $\mathfrak{S}^{(n)} \times F[n]$, которая тождественна над каждым $\{i\} \in F[n]$. Теперь из следствия 2.2.10 получаем, что морфизм $\psi^{\prime(n)}: \mathfrak{S}^{(n)} \rightarrow \mathfrak{S}^{F[n]}$, соответствующий $E^{\prime}$, гомотопен $\psi^{(n)}$ над $\mathfrak{S}^{n+1}$.

4.2.9. Модулярная интерпретация морфизма $\psi^{(n)}$. В обозначениях из 4.2.7 морфизму $\varphi: K \rightarrow \mathfrak{S}^{(n)}$ соответствует диаграмма $\varphi^{*}(\phi)$ левых расслоений над $K$, в то время как композиции $\psi^{(n)} \circ \varphi: K \rightarrow \mathfrak{S}^{F[n]}$ соответствует левое расслоение $\varphi^{*}(E)$. Напомним, что $\mathrm{Cyl}^{d}(\phi) \rightarrow K \times F[n]-$ квазирасслоение и что $E$ - фибрантная замена для $\mathrm{Cyl}^{d}(\phi)$, такая, что $\left.E\right|_{i}=E^{(i)}$ для всех $i$. Поэтому из следствия 3.1.4 вытекает, что $\varphi^{*}(E)-$ фибрантная замена для $\varphi^{*}\left(\mathrm{Cyl}^{d}(\phi)\right)=$ $\mathrm{Cyl}^{d}\left(\varphi^{*}(\phi)\right)$, такая, что $\left.\varphi^{*}(E)\right|_{i}=\varphi^{*}\left(E^{(i)}\right)$ для всех $i$.

4.2.10. Доказательство предложения 2.2.9(c). Согласно 2.2.7, композиции $\mu^{*} \circ \psi^{(n)}: \mathfrak{S}^{(n)} \rightarrow \mathfrak{S}^{F[m]}$ соответствует левое расслоение $\mu^{*}(E) \rightarrow \mathfrak{S}^{(n)} \times$ $F[m]$, которое, как и в 4.2 .9 , является фибрантной заменой для $\mu^{*}\left(\mathrm{Cyl}^{d}(\phi)\right)=$ $\mathrm{Cyl}^{d}\left(\mu^{*}(\phi)\right)$, такой, что $\left.\mu^{*}(E)\right|_{i}=E^{(\mu(i))}=\left.\mathrm{Cyl}^{d}\left(\mu^{*}(\phi)\right)\right|_{i}$ для всех $i$. Далее, морфизму $\psi^{(m)} \circ \mu^{*}: \mathfrak{S}^{(n)} \rightarrow \mathfrak{S}^{F[m]}$ также соответствует фибрантная замена для $\mathrm{Cyl}^{d}\left(\mu^{*}(\phi)\right)$ с аналогичными свойствами. Теперь нужное утверждение вытекает из следствия 2.2.10, как и в 4.2.8.

Остается показать, что $\psi:=\psi^{(n)}$ - гомотопическая эквивалентность над $\mathfrak{S}^{n+1}$.

4.2.11. Редукция. Согласно лемме 1.3.11(a), нужно доказать, что для любого отображения $\eta: M \rightarrow \mathfrak{S}^{n+1}$ отображение $\pi_{0}(\psi / \eta): \pi_{0}\left(\operatorname{Map}_{\mathfrak{S}^{n+1}}\left(M, \mathfrak{S}^{(n)}\right)\right) \rightarrow$ $\pi_{0}\left(\operatorname{Map}_{\mathfrak{S}^{n+1}}\left(M, \mathfrak{S}^{F[n]}\right)\right)$, индуцированное отображением $\psi$, является биекцией. 
Пусть $\eta$ соответствует упорядоченному набору из $n+1$ левых расслоений $H^{(0)}, \ldots, H^{(n)}$ над $M$. Тогда $\varphi \in \operatorname{Hom}_{\mathfrak{S}^{n+1}}\left(M, \mathfrak{S}^{(n)}\right)$ соответствует диаграмме $\varphi: H^{(0)} \stackrel{\varphi_{1}}{\longrightarrow} \cdots \stackrel{\varphi_{n}}{\longrightarrow} H^{(n)}$ над $M$ и $\tau \in \operatorname{Hom}_{\mathfrak{S}^{n+1}}\left(M, \mathfrak{S}^{F[n]}\right)$ соответствует левому расслоению $H \rightarrow M \times F[n]$, такому, что $\left.H\right|_{i}=H^{(i)}$ для всех $i$.

Используя следствие 2.2.10 и п. 4.2.9, видим, что $\psi \circ \varphi \sim \tau$ в $\operatorname{Map}_{\mathfrak{S}^{n+1}}\left(M, \mathfrak{S}^{F[n]}\right)$ тогда и только тогда, когда существует морфизм $\nu: \mathrm{Cyl}^{d}(\varphi) \rightarrow H$ над $M \times$ $F[n]$, такой, что $\left.\nu\right|_{i}:\left.H^{(i)} \rightarrow H\right|_{i}=H^{(i)}$ есть $\operatorname{Id}_{H^{(i)}}$ для всех $i$ и $\nu-$ слабая эквивалентность. Кроме того, в силу п. 4.1.1(е) предположение о том, что $\nu-$ слабая эквивалентность, выполнено автоматически.

4.2.12. Доказательство сюръективности отображения $\pi_{0}(\psi / \eta)$. Нам нужно доказать, что для любого левого расслоения $H \rightarrow M \times F[n]$, такого, что $\left.H\right|_{i}=H^{(i)}$ для всех $i$, существует диаграмма $\varphi$ и морфизм $\nu: \mathrm{Cyl}^{d}(\varphi) \rightarrow H$ над $M \times F[n]$, такие, что каждое отображение $\left.\nu\right|_{i}:\left.H^{(i)} \rightarrow H\right|_{i}=H^{(i)}$ является тождественным. Построим $\varphi$ и $\nu$ индукцией по $n$. Если $n=0$, то $\varphi$ пусто и $\mathrm{Cyl}^{d}(\varphi)=H^{(0)}=H$, так что $\nu=\operatorname{Id}_{H}$ удовлетворяет нужным условиям.

Предположим, что $n>0$. По предположению индукции существует диаграмма $\varphi^{(1)}: H^{(1)} \stackrel{\varphi_{2}}{\longrightarrow} \cdots \stackrel{\varphi_{n}}{\longrightarrow} H^{(n)}$ над $M$ и морфизм $\left.e^{1} \mathrm{Cyl}^{d}(\varphi(1)) \rightarrow H\right|_{e^{1} F[n-1]} \subset H$ над $M \times F[n]$, такие, что $\left.\nu\right|_{i}:\left.H^{(i)} \rightarrow H\right|_{i}=H^{(i)}$ - тождественное отображение для всех $i>0$. В частности, существует морфизм $\nu[1]: H^{(1)} \times e^{1} F[n-1] \rightarrow$ $e^{1} \mathrm{Cyl}^{d}(\varphi(1)) \rightarrow H$ над $M \times F[n]$, такой, что $\left.\nu[1]\right|_{1}=\operatorname{Id}_{H^{(1)}}$.

Поскольку $\mathrm{Cyl}^{d}(\varphi)=\left(H^{(0)} \times F[n]\right) \sqcup_{\left(H^{(0)} \times e^{1} F[n-1]\right)} e^{1} \mathrm{Cyl}^{d}(\varphi(1))$, остается построить морфизм $\varphi_{1}: H^{(0)} \rightarrow H^{(1)}$ над $M$ и морфизм $\nu[0]: H^{(0)} \times F[n] \rightarrow H$ над $M \times F[n]$, такие, что $\left.\nu[0]\right|_{0}=\operatorname{Id}_{H^{(0)}}$ и сужение $\left.\nu[0]\right|_{e^{1} F[n-1]}$ разлагается в композицию $H^{(0)} \times e^{1} F[n-1] \stackrel{\varphi_{1}}{\longrightarrow} H^{(1)} \times e^{1} F[n-1] \stackrel{\nu[1]}{\longrightarrow} H$.

Так как $H \rightarrow M \times F[n]$ - левое расслоение, то включение $H^{(0)}=\left.H\right|_{0} \hookrightarrow H$ расширяется до морфизма $\nu^{\prime}[0]: H^{(0)} \times\left. e^{0} F[1] \rightarrow H\right|_{e^{0} F[1]} \subset H$ над $M \times F[n]$ (по лемме 4.1.4(a)). Обозначим $\left.\nu^{\prime}[0]\right|_{1}: H^{(0)} \rightarrow H^{(1)}$ через $\varphi_{1}$ и определим $\nu^{\prime \prime}[0]: H^{(0)} \times\left. e^{1} F[n-1] \rightarrow H\right|_{e^{1} F[n-1]} \subset H$ как композицию $\nu[1] \circ \varphi_{1}$. Тогда $\nu^{\prime}[0]$ и $\nu^{\prime \prime}[0]$ определяют морфизм $H^{(0)} \times\left(e^{0} F[1] \sqcup_{e^{1} F[0]} e^{1} F[n-1]\right) \rightarrow H$ над $M \times F[n]$, который по лемме 4.1.4(b) можно расширить на всё $H^{(0)} \times F[n]$.

4.2.13. Доказательство инъективности отображения $\pi_{0}(\psi / \eta)$. Зафиксируем левое расслоение $H \rightarrow M \times F[n]$ и рассмотрим все диаграммы $\varphi: H^{(0)} \stackrel{\varphi_{1}}{\longrightarrow}$ $\cdots \stackrel{\varphi_{n}}{\longrightarrow} H^{(n)}$ над $M$, для которых существует морфизм $\nu: \mathrm{Cyl}^{d}(\varphi) \rightarrow H$ над $M \times F[n]$, такой, что каждое отображение $\left.\nu\right|_{j}$ тождественное. Нам нужно доказать, что каждое $\pi_{0}\left(\varphi_{j}\right) \in \pi_{0}\left(\operatorname{Map}_{M}\left(H^{(j)}, H^{(j+1)}\right)\right)$ зависит только от $H$.

Рассмотрим морфизм $\iota_{j}: H^{(j)} \times e^{j} F[n-j] \rightarrow \mathrm{Cyl}^{d}(\varphi)$, построенный в 4.1.2. Тогда композиция $\nu \circ \iota_{j}: H^{(j)} \times e^{j} F[n-j] \rightarrow H$ такова, что $\left.(\nu \circ \iota)\right|_{j}: H^{(j)} \rightarrow H^{(j)}$ есть $\operatorname{Id}_{H^{(j)}}$, в то время как $\left.(\nu \circ \iota)\right|_{j+1}: H^{(j)} \rightarrow H^{(j+1)}$ есть $\varphi_{j}$. Таким образом, остается показать, что каждое $\pi_{0}\left(\nu \circ \iota_{j}\right) \in \pi_{0}\left(\operatorname{Map}_{M \times F[n]}\left(H^{(j)} \times e^{j} F[n-j], H\right)\right)$ зависит только от $H$. Поскольку отображение сужения $\operatorname{Map}_{M \times F[n]}\left(H^{(j)} \times e^{j} F[n-\right.$ $j], H) \rightarrow \operatorname{Map}_{M \times F[n]}\left(H^{(j)} \times\{j\}, H\right)$ является тривиальным расслоением (по лемме 4.1.4(b)), в то время как $\left.\left(\nu \circ \iota_{j}\right)\right|_{j}=\operatorname{Id}_{H^{(j)}}$, нужное утверждение следует из $1.2 .8(\mathrm{~b})$. 


\section{ЛитеРАТУРА}

[1] P. Goerss, J. Jardine, Simplicial Homotopy Theory, Progress in Math., vol. 174, Birkhäuser, Basel, 1999.

[2] D. Kazhdan, Y. Varshavsky, Yoneda lemma for complete Segal spaces II (готовится к печати).

[3] D. Kazhdan, Y. Varshavsky, Yoneda lemma for $(\infty, n)$-categories (готовится к печати).

[4] J. Lurie, Higher Topos Theory, Annals of Math. Studies, vol. 170, Princeton University Press, Princeton, NJ, 2009.

[5] J. Lurie, Higher Algebra, http://www.math.harvard.edu/lurie/.

[6] C. Rezk, A model for the homotopy theory of homotopy theory, Trans. Amer. Math. Soc., 353:3 (2001), 973-1007.

[7] C. Rezk, A Cartesian presentation of weak n-categories, Geom. Topol., 14:1 (2010), 521-571, 14:4 (2010), 2301-2304.

[8] C. Rezk, Fibrations and homotopy colimits of simplicial sheaves, arXiv:math/9811038.

[9] D. Quillen, Homotopical algebra, Lecture Notes in Math., vol. 43, Springer-Verlag, Berlin-New York, 1967.

Institute of Mathematics,

The Hebrew University of Jerusalem

Поступило в редакцию

e-mail:vyakov@math.huji.ac.il

22 января 2014 г.

Institute of Mathematics,

The Hebrew University of Jerusalem

e-mail: kazhdan@math.huji.ac.il 